\title{
Evaluación del impacto del cambio climático sobre una cuenca hidrológica en régimen natural (SE, España) usando un modelo SWAT
}

\author{
Antonio Jodar-Abellan ${ }^{1, *}$, Marcos Ruiz $^{2}$ y Joaquin Melgarejo ${ }^{1}$ \\ ${ }^{1}$ Universidad de Alicante, Instituto Universitario del Agua y las Ciencias Ambientales, \\ Carretera San Vicente del Raspeig s/n, 03690, Alicante, España. \\ ${ }^{2}$ Universidad de Murcia, Instituto Universitario del Agua y del Medio Ambiente, \\ Edificio D, Campus de Espinardo, 30100, Murcia, España. \\ *antonio.jodar@ua.es
}

\section{RESUMEN}

Actualmente, las demandas hídricas se encuentran en continuo crecimiento lo que aumenta la presión, en cantidad y calidad, sobre los recursos hídricos disponibles a nivel global. Asimismo, la reducción proyectada de las aportaciones en régimen natural, consecuencia del cambio climático, supone un nuevo nivel de incertidumbre. Pese a errores todavía existentes, los modelos hidrológicos constituyen herramientas vitales para planificar y gestionar el recurso agua. Su correcta aplicación resulta esencial en áreas con elevado estrés hídrico (regiones áridas y semiáridas). La Demarcación Hidrográfica del Segura (DHS), sureste de España, presenta una de las mayores tasas de escasez y explotación intensiva de recursos hídricos en el contexto europeo y mundial. Este trabajo estima los efectos del cambio climático sobre los principales componentes del balance hídrico en la cuenca alta del río Taibilla (cabecera de la DHS). Dicha cuenca fue escogida al encontrase en régimen natural y ser actualmente primordial en el abastecimiento hídrico de numerosos municipios de la DHS y áreas contiguas. Para evaluar los mencionados efectos se ha implementado el modelo Soil and Water Assessment Tool (SWAT) calibrado con el algoritmo SUFI-2. La calibración y validación se realizaron con dos funciones objetivo (NSE y KGE) en el periodo con caudales observados (1996-2012). Las proyecciones de precipitación y temperatura proceden de los modelos climáticos globales (GCMs) bcc-csm1-1-m, MPI.ESM.MR y bcc. csm1.1, regionalizados con la técnica de Análogos, bajo los escenarios de emisión RCP4.5 y RCP8.5 (periodo 2010-2050). Los resultados del ajuste mejoran con NSE, con respecto a KGE, en las series de calibración y validación. En las próximas décadas, precipitación, escorrentía superficial y recarga de acuíferos muestran tendencias negativas mientras que la temperatura aumenta entre $1.5^{\circ} \mathrm{C}$ y $1.7^{\circ} \mathrm{C}$. Pese a la incertidumbre existente, resulta evidente que las aportaciones disminuirán en la cuenca lo que supondrá un reto en la gestión del recurso agua para las autoridades locales. Los resultados obtenidos pueden ayudar a las autoridades locales en la toma de decisiones respecto a la plani- ficación hidrológica y la lucha contra el cambio climático en la DHS. Así mismo, SWAT como herramienta de conversión lluvia-escorrentía, junto con los modelos y escenarios de cambio climático implementados, resultan válidos en un área de clima subhúmedo y semiárido pudiendo ser aplicados en otras regiones con una climatología similar.

Palabras clave: recursos hídricos; SWAT; SUFI-2; cambio climático; RCPs; GCMs; cuenca alta del Taibilla.

\begin{abstract}
Currently, water demands are in continuous growth which increases the pressure, in quantity and quality, on the water resources available over the world. Likewise, the projected reduction of natural water inputs, due to climate change, represents a new level of uncertainty. Despite existing errors, hydrological models are important tools for planning and managing water resources. Their correct application is essential in areas with high water stress (arid and semi-arid regions). The Segura River basin district (DHS), southeastern Spain, has one of the highest scarcity rates and intensive exploitation of water resources in Europe and the world. This study estimates the effects of climate change on the main components of the water balance in the upper basin of the Taibilla River (the headwater of the DHS). This basin has been chosen because it is in natural regime and it is currently one of the main water supplies in many DHS municipalities and contiguous areas. In order to assess the mentioned effects, we used the Soil and Water Assessment Tool (SWAT) calibrated with the SUFI-2 algorithm. Calibration and validation were performed with two objective functions (NSE and KGE) in the period with availability of discharge data (1996-2012). The precipitation and temperature projections came from the global climate models (GCMs) bcc-csm1-1-m, MPI.ESM.MR and bcc.csm1.1, regionalized with the analogue technique, under the emission scenarios RCP4.5 and RCP8.5 (period 2010-2050). The adjustment results improved with NSE, with respect to KGE, in the calibration and validation series. In the future
\end{abstract}


decades, precipitation, surface runoff and recharge of aquifers would show negative trends while the temperature would increase by between $1.5^{\circ} \mathrm{C}$ and $1.7^{\circ} \mathrm{C}$. In spite of the existing uncertainty, it is evident that natural water inputs will decrease in this river basin. This will be a challenge in the water resources management for local authorities. The obtained results can assist local authorities at decision making regarding hydrological planning and the fight against climate change in the DHS. In addition, SWAT as a rainfall-runoff conversion tool, together with the implemented models and climate change scenarios, are suitable in an area with subhumid and semi-arid climate and can be used in other regions with similar climatology.

Key words: water resources; SWAT; SUFI-2; climate change; RCPs; GCMs; upper basin of the Taibilla River.

\section{INTRODUCCIÓN}

Actualmente, a nivel global, el crecimiento demográfico, el aumento en el nivel de vida, el turismo, las políticas de ordenación del territorio y de uso y gestión del agua, etc., ejercen una presión sobre los recursos hídricos disponibles adicional a la ya existente por parte de la agricultura, la producción energética, los usos industriales y domésticos, y el medio ambiente (Abbaspour et al., 2015; Sánchez-Galiano et al., 2017). Las reservas hídricas, superficiales y subterráneas (agua azul), se encuentran considerablemente amenazadas, en cantidad y calidad, en regiones con elevado estrés hídrico, originado en gran medida por el uso agrícola (Cynthia et al., 2004; Wada et al., 2012; Custodio et al., 2016). En consecuencia, los reservorios de agua dulce han disminuido, lo que acarrea impactos ambientales negativos sobre los ecosistemas asociados (Klove et al., 2014). A su vez, la gestión del agua dulce se encuentra supeditada a cambios de criterio e ideologías políticas, caso por ejemplo de los trasvases entre cuencas en España, que aportan incertidumbre al abastecimiento (Molina y Melgarejo, 2015). Al mismo tiempo, el cambio global aporta un nuevo nivel de incertidumbre en los suministros de agua dulce y en los principales sectores económicos con un uso consuntivo elevado de agua como la agricultura y la energía. Dicha problemática aumentará la inseguridad en las futuras demandas del agua (Green et al., 2011), lo que, junto al continuo aumento en la frecuencia y duración de sequías e inundaciones (Wanders y Van-Lanen, 2015; Pérez-González et al., 2017), provocará que las sociedades amplíen su vulnerabilidad ante un extenso elenco de riesgos asociados con el suministro inadecuado del agua en cantidad y/o calidad (UNESCO, 2012).

El cambio global abarca variaciones climáticas a nivel espacial y temporal, y cambios derivados de los procesos terrestres, incluyendo actividades humanas que afectan al medio ambiente, y como tales, el cambio global proyectado incluye los sistemas de recursos hídricos (Green et al., 2011). En los últimos años, el cambio global (cambio climático, de usos del suelo, etc.) ha sido plenamente demostrado (IPCC, 2013). La influencia antrópica sobre el medio ha alterado el clima de los últimos 25 años, de modo que las modificaciones registradas a escala planetaria no podrían atribuirse exclusivamente a las anomalías y variabilidad natural del sistema climático (Allen et al., 2000; Bindoff et al., 2013). Actualmente, se consideran cuatro escenarios de variación climática para los próximos decenios: los RCP (Representative Concentration Pathways), denominados RCP2.6, RCP4.5, RCP6.0 y RCP8.5 según la magnitud del forzamiento radiativo que aportan al sistema climático natural. Por ello, los resultados de los modelos climáticos globales (GCMs) y regionales (RCMs) varían en función del forzamiento introducido (IPCC, 2013).

Los modelos matemáticos aplicados a la hidrología (modelos hidrológicos) constituyen herramientas vitales con las que planificar el uso y gestión sostenible de los recursos hídricos, permitiendo satisfacer, en lo posible, las demandas agrícolas, urbanas, industriales y ambientales (Praskievicz y Chang, 2009; Al Kuisi y El-Naqa, 2013; Abbaspour et al., 2015). En las últimas décadas, debido en parte a los desfavorables escenarios de cambio global, sequías y escasez, la relación entre estos modelos y la hidrología se ha incrementado (Neitsch et al., 2011; Cabezas, 2015). Hoy en día, el número y tipología de modelos desarrollados es elevada. Cabezas (2015) establece 16 tipologías de modelos hidrológicos entre las que existen además tipologías mixtas. Pese a dicha diversidad, tres son las características básicas para clasificar los modelos: i) su finalidad u objetivo, es decir, las variables cuyo comportamiento se pretende modelar; ii) su escala, resolución o parametrización espacial (modelos agregados, semidistribuidos y distribuidos); iii) su escala o alcance temporal (modelos de episodio, y de simulación continua: Praskievicz y Chang, 2009; Cabezas, 2015).

La citada conexión entre los escenarios de cambio global y los modelos hidrológicos ha originado en las últimas décadas numerosos estudios científicos (Cynthia et al., 2004; Praskievicz y Chang, 2009). A nivel global, se identifica un consenso generalizado en la repercusión negativa del cambio global sobre los principales elementos del balance hídrico como reducción de la humedad del suelo, de la escorrentía total (superficial, hipodérmica y subterránea), etc., aunque existen notorias discrepancias en la magnitud de dichos cambios según la región estudiada (Green et al., 2011; Klove et al., 2014; Senent-Aparicio et al., 2017). En España, por ejemplo, se estima que las aportaciones fluviales disminuirán entre un 5 y un 15\% en los próximos 20 años, cuyos efectos serán especialmente visibles en primavera y verano (CEDEX, 2012). Las regiones áridas y semiáridas, como el sureste español, representan un reto en la investigación científica al respecto dado que se caracterizan por una marcada variabilidad pluviométrica traducida, en los modelos de conversión lluvia-escorrentía, en una elevada fluctuación de los caudales aforados dificultando enormemente la consecución de dichos análisis. Son frecuentes los periodos de sequías donde las formaciones de acuíferos suponen la única fuente de suministro hídrico "de origen estrictamente natural", al tiempo que aportan el caudal base en los cursos fluviales (Senent-Alonso y García-Aróstegui, 2014; Aguilar-García y Ortega-Guerrero, 2017). La importancia de los acuíferos en el sureste español ha sido plasmada en diversos estudios de cambio global e hidrogeología (Molina et al., 2013; Touhami et al., 2015; Moutahir et al., 2017) en alguno de los cuales se identifican incluso aumentos en las tasas de recarga en función del GCM y RCM implementado (Pulido-Velázquez et al., 2015). No obstante, existe una escasez notable de estudios equivalentes realizados a escala de cuenca hidrológica y balance hídrico.

En la Demarcación Hidrográfica del río Segura (DHS), donde se localiza el ámbito de actuación de este estudio (cuenca alta del río Taibilla), destaca únicamente Senent-Aparicio et al (2017) en la cuenca del río Madera. Al igual que en dicho estudio, en este trabajo se implementó el modelo hidrológico SWAT (Soil and Water Assessment Tool) desarrollado inicialmente en Arnold et al. (1998). Esta herramienta, ampliamente contrastada en estudios de hidrología y cambio climático a nivel global (Gassman et al., 2007, 2014), fue seleccionada para representar el comportamiento hidrológico de la cuenca analizada dado que permite representar la variabilidad natural intrínseca (diaria o subdiaria) a las regiones semiáridas (Cabezas, 2015). Así mismo, el modelo realiza análisis geográficos espacialmente desagregados de un modo aceptable, posibilitando, entre otros aspectos, la evaluación de prácticas agrícolas sobre la calidad de los recursos hídricos a escala de cuenca (Winchell et al., 2013). Ello resulta esencial dado que el sureste español se encuentra sometido a elevadas demandas agrícolas (Klove et al., 2014; Custodio et al., 2016). 
El objetivo del presente trabajo es analizar el impacto del cambio climático sobre los recursos hídricos de la cuenca alta del río Taibilla. Para ello, en primer lugar, a partir de mediciones del caudal a la salida de las subcuencas (periodo 1996-2012), se aplicó el algoritmo SUFI-2 con el que calibrar, validar y analizar la sensibilidad del modelo SWAT, utilizando dos funciones objetivo diferentes, la eficiencia de NashSutcliffe (NSE) y la eficiencia de Kling-Gupta (KGE). Seguidamente, se incorporaron en SWAT las proyecciones de tres GCMs mediante la regionalización estadística por Análogos (periodo 2010-2050). Los escenarios de cambio climático, seleccionados para dichos GCMs, fueron el RCP4.5 y el RCP8.5. Los resultados del estudio pueden proporcionar a las autoridades locales información útil en la toma de decisiones respecto a la planificación hidrológica y la lucha contra el cambio climático en la DHS. Además, las herramientas implementadas resultan válidas en un área de clima subhúmedo y semiárido pudiendo ser por ello aplicadas en otras regiones del globo con una climatología similar. Especialmente relevante es el análisis comparativo entre NSE y $K G E$, con frecuencia no realizado en estudios científicos similares, dado que influye considerablemente en la calibración del modelo y, por ende, en el correcto ajuste de los caudales simulados a los observados, los cuales presentan a menudo elevadas fluctuaciones en regiones áridas y semiáridas (Cabezas, 2015). Asimismo, el estudio muestra una metodología novedosa en cuanto a la selección de los GCMs y RCMs, acoplados en SWAT, habitualmente no justificada en estudios equivalentes.

\section{ÁREA DE ESTUDIO}

La cuenca del río Taibilla se localiza en la cabecera de la DHS (sureste de España). Con una superficie de $633 \mathrm{~km}^{2}$, representa uno de los afluentes más relevantes del río Segura. Se trata de una cuenca de montaña mediterránea perteneciente a la zona Subbética de las cordilleras Béticas, con una altitud situada entre los $640 \mathrm{~m}$ y los $2080 \mathrm{~m}$ (Quiñonero-Rubio et al., 2013). El embalse del Taibilla, ubicado en el sector central de la cuenca, es considerado la desembocadura (salida) de la cuenca alta del río Taibilla, la cual constituye el área de estudio en este trabajo (Figura 1). Dicha cuenca de cabecera cubre un área de $315 \mathrm{~km}^{2}$ sobre los municipios de Moratalla y Nerpio. El embalse supone el punto situado a menor cota $(940 \mathrm{~m})$, mientras que Las Cabras con $2080 \mathrm{~m}$ (Sierra Las Cabras) es el pico más alto. Las Cabras y los restantes picos de la cuenca se encuentran nevados únicamente durante los principales meses de invierno (AYESA, 2010a).

El clima se clasifica como mediterráneo subhúmedo en transición al semiárido. Presenta una marcada estacionalidad con primaveras y otoños lluviosos, y veranos secos (Boix-Fayos et al., 2007). De acuerdo a los registros facilitados por la Agencia Estatal de Meteorología (AEMET), la precipitación media anual alcanza los $585 \mathrm{~mm}$ y la temperatura media anual los $12^{\circ} \mathrm{C}$. La litología dominante se compone de calizas en zonas montañosas y margas en valles, junto con diversos afloramientos de dolomías, margocalizas y areniscas. Todos los materiales datan del Cretácico, Oligoceno y Mioceno. Los leptosoles réndsicos y líticos suponen los tipos de suelo dominantes, con espesores variables según la zona aunque siempre menores a $50 \mathrm{~cm}$ (IGME, 2003; HWSD, 2017).

La red de drenaje se articula en dos cauces con caudal permanente: el río Taibilla y la rambla de la Rogativa, que presentan numerosos afluentes y ramblizos de menor entidad. Ambos desembocan en el embalse del Taibilla. Asimismo, se localizan cuatro Masas de Agua Subterránea (MASub) incluidas total o parcialmente bajos los límites de la cuenca: Fuente Segura-Fuensanta, Taibilla, Anticlinal de Socovos y Caravaca. Estas MASub albergan 18 acuíferos (Figura 2). CHS (2015a, 2015c, 2015i) indica que las MASub no muestran actualmente alteraciones significativas del régimen natural y que poseen buen estado cuantitativo y químico. Por ello no presentan prórrogas ni objetivos medioambientales menos rigurosos de los establecidos en la Directiva Marco del Agua (DMA, 2000) para los horizontes 2021 y

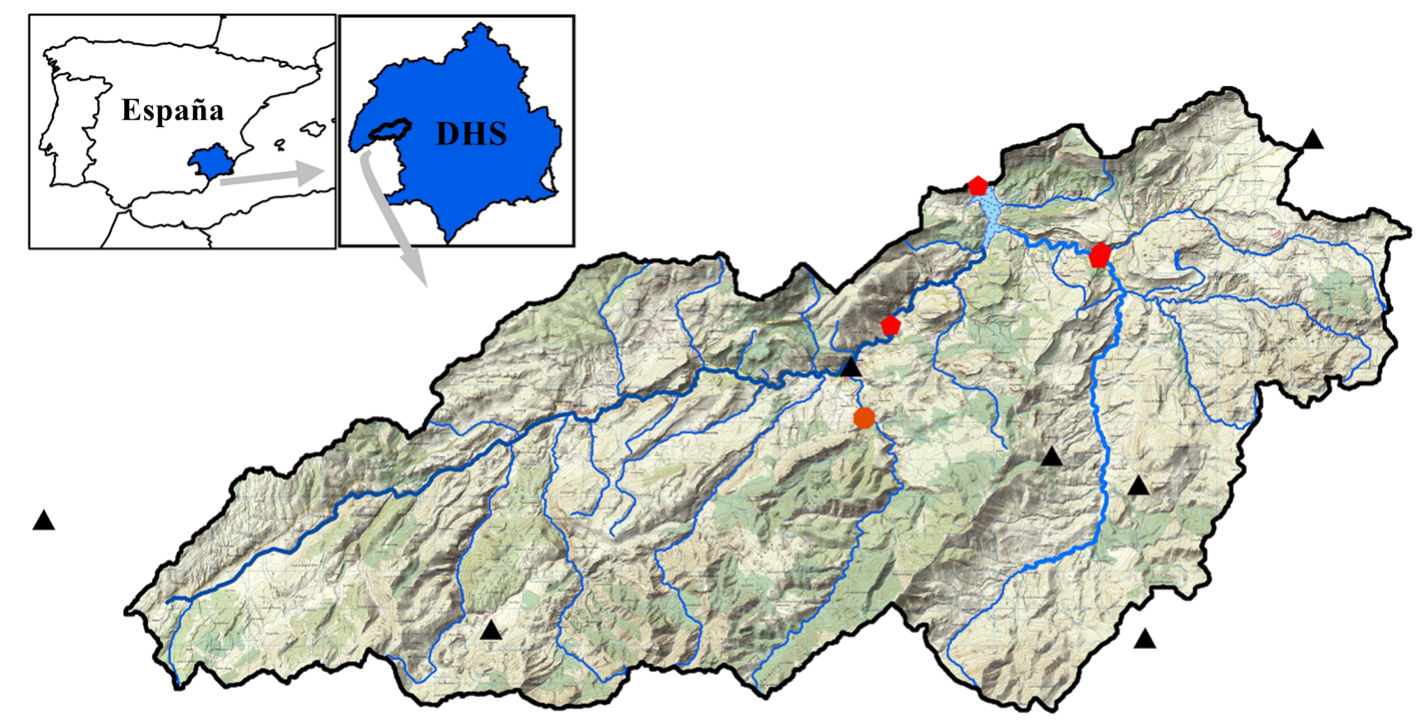

\section{Leyenda}

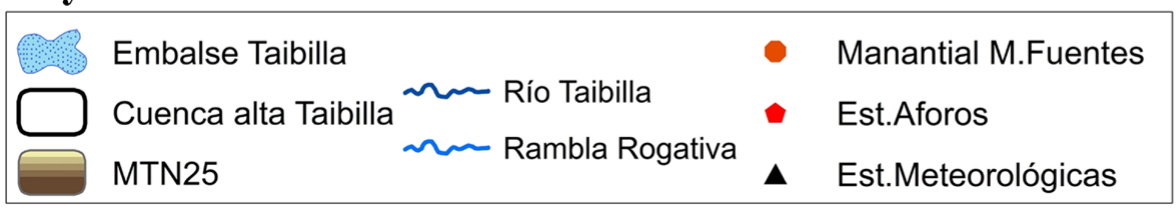

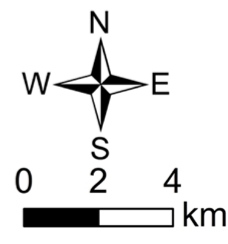

Figura 1. Localización de la cuenca alta del río Taibilla. 


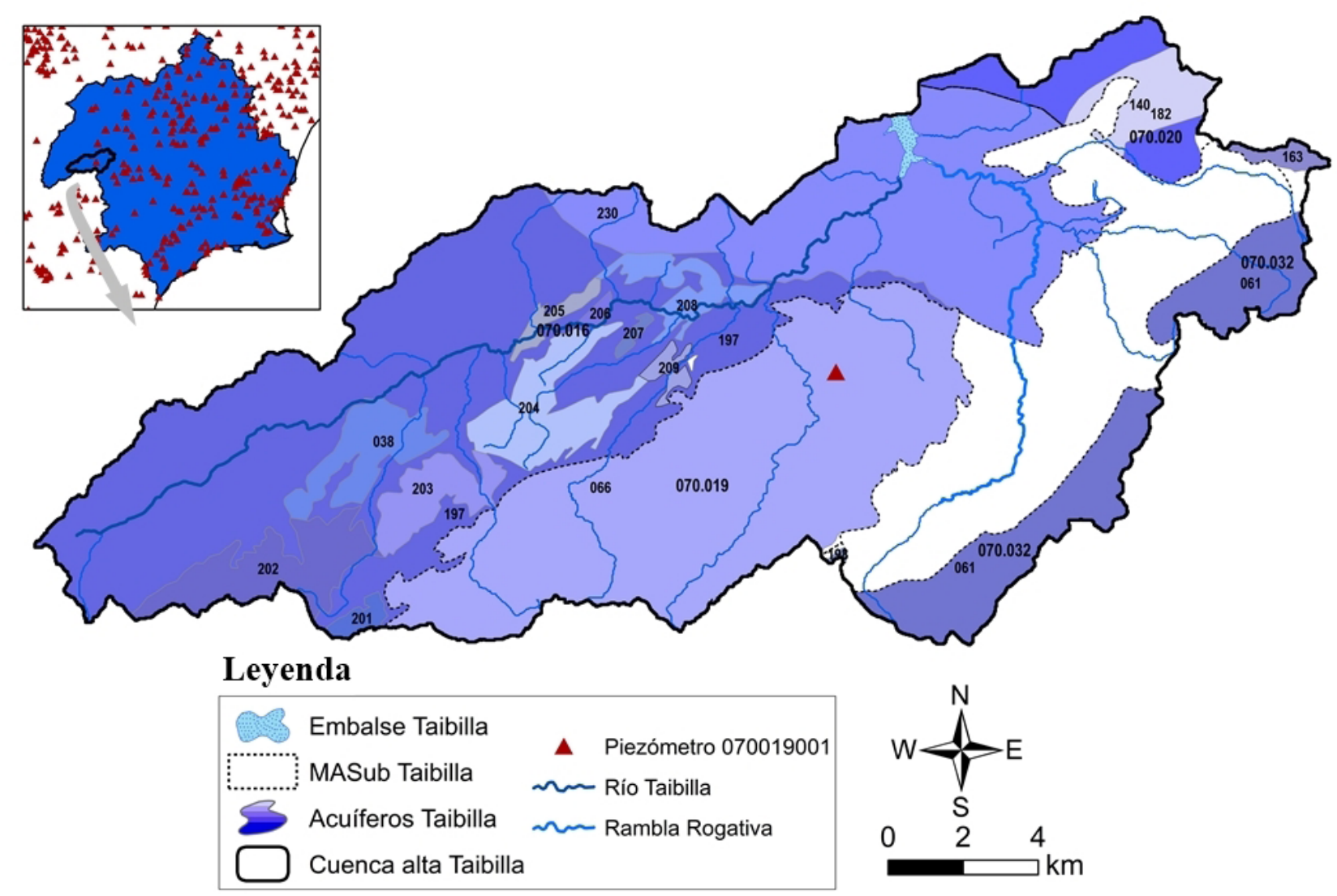

Figura 2. Red piezométrica oficial (arriba), MASub y acuíferos en la cuenca.

2027. A efectos de la modelación hidrológica, destaca la MASub Taibilla y el acuífero Taibilla incluido en ésta. El acuífero supone el principal aporte subterráneo que recibe el embalse. Las surgencias del mismo son recogidas en el único manantial oficialmente inventariado en la cuenca: manantial Molino de las Fuentes (Senent-Alonso y GarcíaAróstegui, 2014; CHS, 2015b). A partir de los registros facilitados por el Centro de Estudios y Experimentación de Obras Públicas (CEDEX) y por AYESA (2010b), en el periodo 01/01/1996-31/12/2012, se estima que las aportaciones totales al embalse alcanzan los $13.3 \mathrm{hm}^{3} /$ año, divididas en la componente superficial e hipodérmica $\left(7.6 \mathrm{hm}^{3} /\right.$ año del río Taibilla y la rambla de la Rogativa) y la subterránea $\left(4.9 \mathrm{hm}^{3} / \mathrm{año}\right)$.

Desde 1950, la cuenca ha sufrido un intenso abandono de las zonas agrícolas lo que ha alterado la estructura del paisaje y los usos del suelo. Ello ha provocado un incremento de las zonas boscosas. Dicha revegetación no se debe únicamente a procesos naturales dado que, desde 1970, se han desarrollado en la cuenca proyectos de restauración hidrológico-forestal que incluían construcción de diques de retención de sedimentos en cauces, reforestación de cabeceras de las subcuencas, etc. (AYESA, 2010a; Quiñonero-Rubio et al., 2013). Estos cambios afectaron a la dinámica de flujos de agua y sedimentos dentro de la cuenca al reducir los caudales aforados y el aporte de sedimentos desde las laderas (Boix-Fayos et al., 2007, 2008). El territorio de la cuenca actualmente destinado a usos agrícolas se incluye en la Unidad de Demanda Agraria "Regadíos aguas arriba del Taibilla" con tan solo $2.22 \mathrm{~km}^{2}$ de superficie y una demanda bruta de agua de $1.55 \mathrm{hm}^{3} /$ año destinados al cultivo de tubérculos como patata (46\%) y almendros (45\%). Recibe recursos hídricos principalmente de la aportación superficial del tramo inicial del río Taibilla no siendo una zona de riego del trasvase Tajo-Segura (CHS, 2015e, 2015h).
El incremento de masa boscosa y de vegetación natural ha favorecido la proliferación en la cuenca de hábitats declarados de interés comunitario por la Directiva Hábitats (DOCE, 1992) y preservados bajo diferentes figuras de protección. La zona presenta actualmente numerosas reservas naturales, endemismos ibéricos, cinco espacios incluidos en la Red Natura2000, una zona de especial protección para las aves (ZEPA) y cuatro lugares de interés comunitario (LIC). Así mismo, el embalse del Taibilla se encuentra propuesto como humedal (Guerrero et al., 2011; CHS, 2015f, 2015g). Dichos espacios muestran conexiones hídricas con determinados acuíferos de la cuenca a través de afloramientos permeables (CHS, 2015g). Junto con las aguas superficiales y subterráneas, el buen estado de estos ecosistemas asociados se debe en parte al escaso desarrollo agrícola, urbano e industrial de la cuenca. Únicamente se identifican tres núcleos de población, con menos de 1300 habitantes en total, los cuales forman parte de las Unidades de Demanda Urbana "MCT-Noroeste y Centro" y "Cabecera del Segura". Ambas demandas son abastecidas parcialmente con recursos hídricos de la cuenca (AYESA, 2010a; CHS, 2015d).

En virtud de lo anterior, se considera que la cuenca alta del Taibilla se halla próxima al régimen natural, condición indispensable para cumplir los objetivos del estudio. Asimismo, desde los años 30 del siglo XX, la Mancomunidad de los Canales del Taibilla (MCT) gestiona los recursos de esta cuenca. Hoy en día, la MCT se cataloga jurídicamente como un organismo público-estatal, de uso y suministro hídrico. La MCT abastece a numerosos municipios de las provincias de Murcia (44), Alicante (35) y Albacete (dos). Las fuentes de suministro que utiliza proceden del trasvase Tajo-Segura y extraordinarios (57.4\%), de la desalación de agua marina (3.4\%) y de los aportes, de origen superficial y subterráneo, almacenados en el embalse del río Taibilla 
(39.2\%). Aunque los mencionados porcentajes varían en función del año hidrológico considerado (MCT, 2014; Melgarejo y Molina, 2017), resulta evidente la importancia de la cuenca en el suministro hídrico de numerosas demandas urbanas y agrícolas de la DHS y áreas aledañas.

\section{METODOLOGÍA}

\section{Descripción del Modelo}

El software en código abierto Soil and Water Assessment Tool (SWAT) es un modelo lluvia-escorrentía, de parámetros semidistribuidos, capaz de simular numerosos procesos físicos a escala temporal continua (anual, mensual, diaria y subdiaria). Su objetivo principal es predecir la producción de agua y sedimentos en cuencas hidrográficas, así como el impacto de prácticas de gestión agrícola sobre la calidad del agua (nutrientes y pesticidas). Presenta una precisión razonable en cuencas de gran tamaño, con variedad en el relieve, en tipos y usos del suelo. Su elevada resolución espacial permite que sea implementado tanto a escala continental como de cuenca hidrológica (Arnold et al., 1998, 2012). No obstante, el uso de SWAT a gran escala requiere simplificaciones importantes. Gassman et al. (2007) y Abbaspour et al. (2015) muestran múltiples aplicaciones de SWAT a escala continental y de cuenca en Europa, África, Asia y EEUU. La componente hidrológica de SWAT permite calcular los elementos del balance hídrico y, en consecuencia, los recursos hídricos (agua azul, verde, etc.) a nivel incluso de subcuenca. La fase terrestre del ciclo hidrológico es simulada con base en la siguiente ecuación del balance hídrico (Ecuación 1).

$$
S W_{t}=S W_{0}+\sum_{t=1}^{t}\left(R_{\text {day }}-Q_{\text {surf }}-E_{a}-w_{\text {seep }}-Q_{g w}\right)
$$

Donde $S W_{t}$ y $S W_{o}$ son el contenido final e inicial de agua en el suelo, $R_{d a y}$ es la precipitación, $Q_{\text {surf }}$ es la escorrentía superficial, $E_{a}$ la evapotranspiración, $w_{\text {seep }}$ el agua que percola en la zona vadosa desde el perfil del suelo, y $Q_{g w}$ es el flujo de retorno. Las variables se expresan en $\mathrm{mm}$ de $\mathrm{H}_{2} \mathrm{O}$ para el día, $i$, y el tiempo, $t$, en días (Neitsch et al., 2011).
En SWAT, cada cuenca hidrológica es dividida en subcuencas a partir de las coberturas disponibles para el relieve del terreno y los cursos fluviales. A su vez, cada subcuenca se subdivide en polígonos, irregulares y de tamaños variables, denominados Unidades de Respuesta Hidrológica (HRU por sus siglas en inglés). Las HRU resultan de la superposición de un mismo tipo de suelo, uso de suelo y pendiente en una subcuenca dada. Suponen el principal avance de SWAT con respecto a otros modelos hidrológicos similares (Winchell et al., 2013). Dicha ventaja permite que, en SWAT, la simulación hidrológica en la fase terrestre no se realice únicamente a nivel de subcuenca sino también a escala de HRU (Arnold et al., 2012).

Al margen de la componente hidrológica, el desarrollo actual del modelo permite que sea utilizado en: la toma de decisiones frente a alternativas de gestión de recursos hídricos; evaluación de contaminación por fuentes no puntuales incluso en grandes cuencas fluviales; predecir el impacto agrícola sobre sedimentos, compuestos químicos y recursos hídricos de cuencas con diferentes condiciones de gestión, tipos y usos de suelo durante largos períodos de tiempo (Neitsch et al., 2011; Abbaspour et al., 2015).

\section{Datos de entrada en SWAT}

El modelo fue implementado como una extensión del software ArcGIS 10.2.2 denominada ArcSWAT 2012.10.2.18 (Arnold et al., 2012; Winchell et al., 2013), en adelante SWAT. La delimitación de la cuenca alta del río Taibilla, y de las subcuencas de éste, se realizó a partir del Modelo Digital de Elevación del Terreno (MDE), con resolución de 25 m x $25 \mathrm{~m}$, propiedad del Centro Nacional de Información Geográfica (CNIG, 2017). Junto con el MDE, se agregó a SWAT la cobertura de la red hidrográfica disponible en la Confederación Hidrográfica del Segura (CHS, 2017). La salida de la cuenca se ubicó en la estación de aforo 7017 localizada en el embalse del Taibilla. A partir de lo anterior, SWAT delimitó la cuenca alta del Taibilla con $315 \mathrm{~km}^{2}$ distribuidos en 25 subcuencas (Figura 3).

En la elaboración de las HRU se empleó el Mapa Nacional de Usos del Suelo del Proyecto Corine Land Cover (CLC2006) disponible en
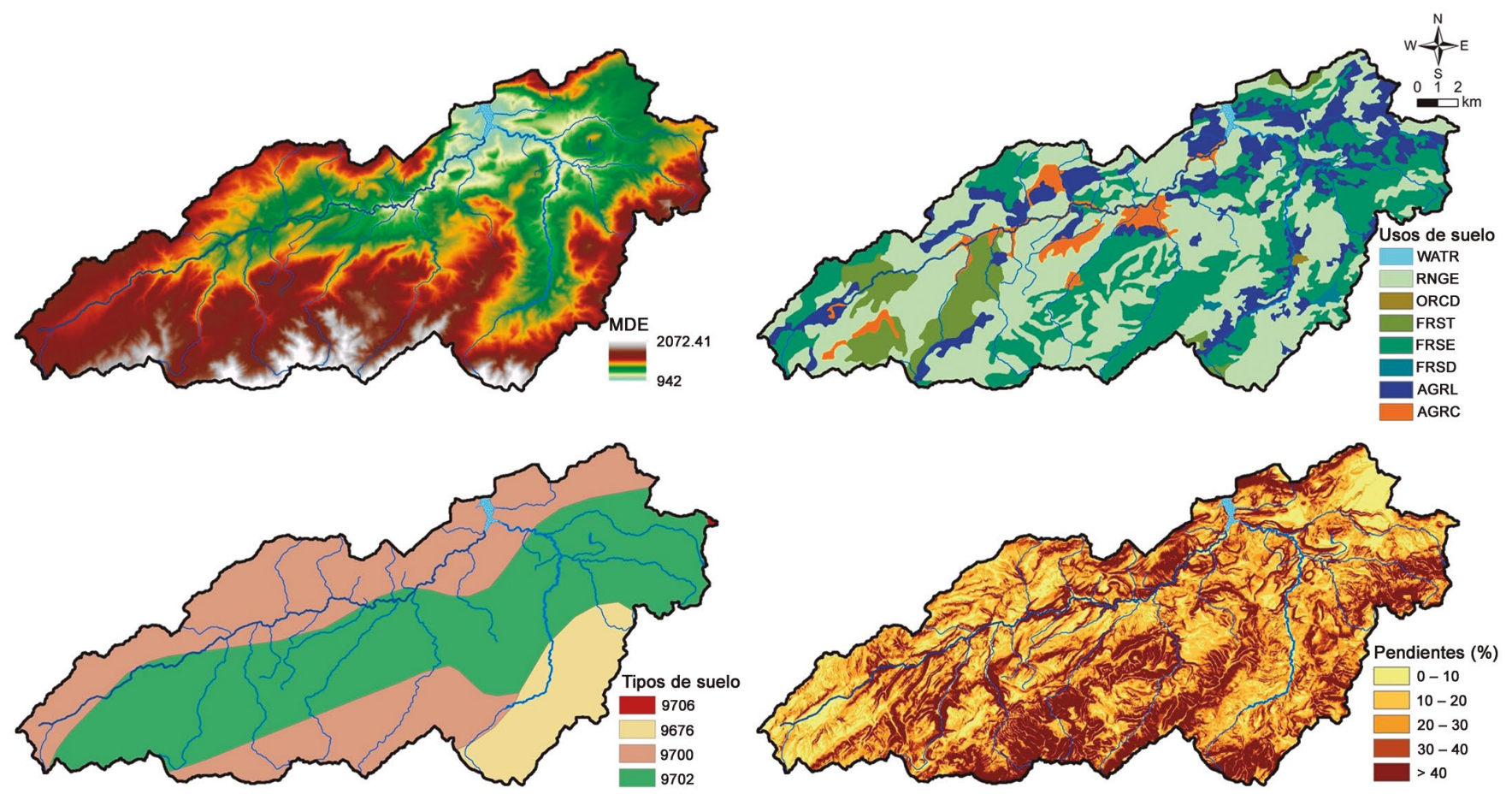

Figura 3. Coberturas del área de estudio en SWAT: relieve (a); usos de suelo (b); edafología (c); clases de pendiente (d). 
CNIG (2017) a escala 1:100000. Los códigos de uso de CLC2006 fueron reclasificados a los de SWAT utilizando la metodología descrita por Bobal et al. (2010) y El-Sadek e İrvem (2014). La edafología de la cuenca se extrajo de la base de datos Harmonized World Soil Database (HWSD, 2012, 2017), a escala 1:1000000, que recoge las unidades de suelo (códigos) establecidas en FAO (1990) y WRB (2014). A partir de la metodología de Gomariz-Castillo (2015), los códigos de suelo de HWSD (2017) fueron incluidos en la base de datos de SWAT. La pendiente fue dividida en cinco clases $(0 \%-10 \%, 10 \%-20 \%, 20 \%-30 \%$, $30 \%-40 \%$ y $>40 \%)$. Dichos umbrales se fijaron ya que la cuenca presenta tramos altos, de cabecera o de alta montaña, con pronunciadas pendientes en las laderas vertientes al cauce del río (AYESA, 2010a; Quiñonero-Rubio et al., 2013). Los terrenos con pendientes suaves quedan relegados casi por completo al cauce del río Taibilla y sus arroyos (Figura 3).

A partir de las coberturas mostradas (Figura 3) se obtuvieron 769 HRU. Debido al gran número de HRU generadas, SWAT se comporta como un modelo de parámetros distribuidos. El tamaño de cada HRU resulta por tanto menor que la superficie de las celdas en las que se divide el territorio, situada habitualmente entre $12 \mathrm{~km}^{2}$ y $5 \mathrm{~km}^{2}$ (Cabezas, 2015). Por ello, los parámetros hidrológicos que caracterizan a la cuenca varían espacialmente en cada punto (HRU < celda).

Tras elaborar las HRU, se incorporaron en SWAT las series diarias de precipitación, temperatura máxima y mínima de las estaciones meteorológicas localizadas en las inmediaciones y en el interior de la cuenca (periodo 01/01/1944 - 30/04/2016). Los citados registros fueron facilitados por AEMET, y posteriormente tratados en el software R3.3.2 $(R, 2013)$ para incluir valores ausentes, años bisiestos, etc. A partir de estas series, se seleccionó el método de Hargreaves (Hargreaves et al., 1985) para calcular la Evapotranspiración Potencial (ETP). Por otro lado, SWAT asigna al centroide de cada subcuenca, calculado previamente, los registros de la estación más cercana (Winchell et al., 2013). La distribución de las ocho estaciones escogidas en la cuenca resulta homogénea (Figura 1), permitiendo con ello que el modelo realice una asignación diferenciada y adecuada, de las variables precipitación y temperatura, a los distintos grupos de subcuencas. Gracias a la distribución que presentan dichas estaciones, no se incluyeron en
SWAT las denominadas bandas de elevación con las que aplicar un gradiente a las series de precipitación y temperatura en función de la altitud (Winchell et al., 2013).

\section{Calibración, validación y análisis de sensibilidad de SWAT}

Los resultados (simulaciones) de los modelos hidrológicos deben ser corregidos con datos medidos. Se restringe así la incertidumbre de las simulaciones pudiendo representar, aunque simplificado, el comportamiento de una cuenca hidrográfica (Gupta et al., 2009; Abbaspour et al., 2015). Este procedimiento se realiza con las técnicas de calibración, validación y análisis de sensibilidad (Moriasi et al., 2007). La calibración evalúa los parámetros del modelo comparando sus predicciones, para un conjunto de condiciones de contorno asumidas, con datos observados para las mismas condiciones. En la validación se lanza el modelo utilizando parámetros determinados en calibración, de modo que éste sea capaz de realizar simulaciones próximas a los datos reales de la serie temporal destinada a validar el modelo (Krause et al., 2005; Moriasi et al., 2007). En SWAT, la calibración y validación, pueden realizarse con medidas de caudal, nitratos, fosfatos, etc. (Abbaspour, 2015; Abbaspour et al., 2015). En el presente estudio, se escoge la variable caudal a la salida de las subcuencas para calibrar y validar SWAT. El análisis de sensibilidad determina el cambio en las salidas del modelo con respecto a las entradas (parámetros) de éste. Es un proceso necesario para identificar parámetros clave y la precisión de los parámetros requerida en calibración (Ma et al., 2000; Moriasi et al., 2007).

\section{Descripción del algoritmo SUFI-2 en SWAT-CUP}

La optimización (calibración) y el análisis de sensibilidad de SWAT se realizaron con el algoritmo SUFI-2 (Sequential Uncertainty Fitting version-2) incluido en el software SWAT-CUP2012 (Abbaspour, 2015). En SUFI-2 la calibración se realiza con una serie de iteraciones que incluyen numerosas simulaciones. Cada nueva iteración es alimentada con los resultados de la anterior. Esto persigue aproximar (optimizar) la variable simulada, caudal a la salida de las subcuencas, a la observada. Los resultados de las iteraciones son un conjunto de valores (rangos) asignados a los parámetros que representan los procesos hidrológicos,

Tabla 1. Estimadores empleados en la bondad del ajuste.

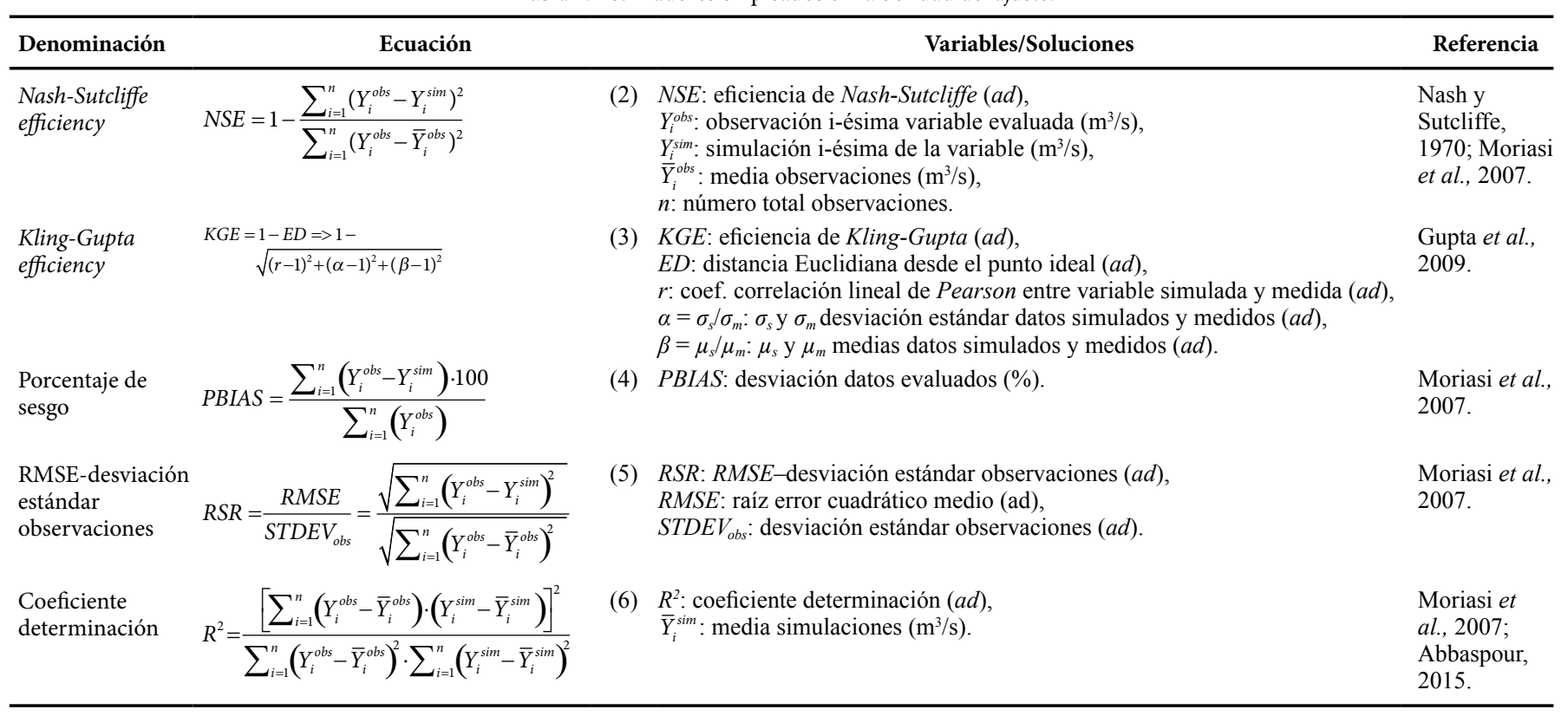


las características físicas y la dinámica de cada cuenca hidrográfica. Cada nueva iteración presenta intervalos (rangos) de los parámetros recursivamente más próximos a su valor real. Ello pretende acotar la incertidumbre existente en los rangos iniciales de los parámetros, dado que a menudo no se dispone de mediciones de estos (Abbaspour, 2015; Abbaspour et al., 2007). Así, a partir de mediciones de caudales, introducidas en SUFI-2, éste proporciona iteración tras iteración mayor exactitud en los rangos de los parámetros de cada área de estudio. Dicho procedimiento se denomina Modelización Hidrológica Inversa (Beven y Binley, 1992; Abbaspour et al., 2004, 2007). En el estudio se escogen 14 parámetros hidrológicos condicionados por los métodos de cambio R-relative y V-replaces (Abbaspour, 2015). Así mismo, en cada iteración, SUFI-2 dispone de estimadores (estadísticos) para evaluar la bondad del ajuste entre el caudal simulado (Qsim) y el observado (Qobs: Tabla 1, ecuaciones 2 a 6). En consecuencia, se escoge la simulación más próxima al caudal observado, es decir, la que presenta mejores resultados en dichos estimadores. Por ello, se consideran óptimos los valores de los parámetros obtenidos en dicha simulación. La validación por su parte se implementó en R3.3.2 para contrastar la serie de Qsim con Qobs mediante los citados estimadores.

Por otro lado, para iniciar la calibración, SUFI-2 requiere escoger un estadístico como función objetivo. La selección de esta función depende de los objetivos del estudio, de las variables a calibrar y de los parámetros hidrológicos a optimizar. Asimismo, la elección de la función afecta a los resultados de la calibración (parámetros hidrológicos, variable simulada-optimizada, etc. Abbaspour et al., 2004). En el estudio se han realizado cinco iteraciones (de 2000 simulaciones) con NSE y otras cinco con KGE. NSE es el estadístico más usado en modelación hidrológica como función objetivo dado que incrementa el rendimiento (ajuste) entre simulaciones y observaciones. Sin embargo, en ocasiones presenta errores por divergencia entre la serie simulada y la observada (Nash y Sutcliffe, 1970). KGE posee un comportamiento similar a NSE, aunque proporciona un mejor ajuste del modelo en caso de disponer de mediciones con numerosos picos (caudales punta). Corrige así el sesgo generado por el modelo entre series, observadas y simuladas, con caudales punta (Gupta et al., 2009; Gomariz-Castillo, 2015).

El análisis de sensibilidad, implementado en SUFI-2 tras la calibración, indica la relación entre la función objetivo y los parámetros hidrológicos seleccionados, cuyos valores cambian según presenten mayor o menor sensibilidad a la función. Los parámetros con mayor sensibilidad (significativa) poseen un $P$-valor $\leq 0.05$. Un número elevado de parámetros con sensibilidad significativa indica una correcta elección de la función objetivo y de los parámetros. No obstante, a medida que las iteraciones son ejecutadas el número de parámetros significativos disminuye. Por ello, los parámetros significativos en las últimas iteraciones serán aquellos cuyo cambio afectará en mayor medida a los resultados del modelo (Abbaspour, 2015; Abbaspour et al., 2015). Información detallada respecto a los procedimientos de calibración, validación y análisis de sensibilidad en el algoritmo SUFI-2 puede ser encontrada en Abbaspour (2015).

\section{Datos de entrada en SUFI-2}

La cuenca alta del río Taibilla posee cuatro estaciones de aforo (Figura 1). En la calibración y validación de SWAT únicamente se consideraron los registros de caudales de la estación 7017 (periodo 01/01/1996-31/12/2012) facilitados por el CEDEX. Las restantes estaciones presentan series obsoletas (mediciones de 1930 a 1950) y con numerosos valores ausentes. La estación 7017, localizada en el embalse del Taibilla (salida de la cuenca), registra las reservas y salidas, a nivel mensual, del embalse. Dado que los embalses constituyen elementos de regulación de caudales, otorgando un régimen hídrico alterado, se realizó una operación de balance hídrico para restituir el régimen natural de los caudales en la salida de la cuenca. A partir de las reservas y salidas se obtuvieron las entradas medias mensuales en el embalse (Ecuación 7).

$$
E_{n}=\left(R_{n}-R_{n-1}\right)+S_{n}+E v_{n}
$$

Donde $E_{n}$ son las entradas al embalse en el mes actual $\left(\mathrm{m}^{3} / \mathrm{s}\right)$, $R_{n}$ las reservas del embalse en el mes actual $\left(\mathrm{m}^{3} / \mathrm{s}\right), R_{n-1}$ las reservas en el mes anterior $\left(\mathrm{m}^{3} / \mathrm{s}\right), S_{n}$ las salidas en el mes actual $\left(\mathrm{m}^{3} / \mathrm{s}\right)$, y $E v_{n}$ las pérdidas por evaporación en el mes actual $\left(\mathrm{m}^{3} / \mathrm{s}\right)$.

Las pérdidas por evaporación $\left(\mathrm{m}^{3} / \mathrm{s}\right)$ se calcularon a partir del área inundada del embalse (763009 $\mathrm{m}^{2}$ variable según el año hidrológico) y de los registros de evaporación en el embalse $(\mathrm{mm} / \mathrm{mes})$ disponibles en CHS (2015h). Dichas pérdidas afectan considerablemente al balance debido a la climatología del área de estudio, con clima semiárido, registrando en el embalse una evaporación media situada entre 52.5 $\mathrm{mm} / \mathrm{mes}$ y $272.4 \mathrm{~mm} / \mathrm{mes}$ (CHS, 2015h). Las pérdidas del embalse por filtración se estiman despreciables debido a la formación del vaso del embalse compuesta por materiales impermeables (arcillas y margas) cuya acción se une al sedimento acumulado en el lecho del embalse (CHS, 2015c).

Las entradas mensuales al embalse, obtenidas por balance (periodo 01/01/1996-31/12/2012), suponen la aportación total que éste recibe (superficial y subterránea). Los dos registros ausentes identificados en dicha serie fueron contrastados y completados con el aporte superficial del embalse durante el periodo 01/01/1996-31/12/2010 (AYESA, $2010 \mathrm{~b}$ ) y con el caudal registrado en el Manantial Molino de las Fuentes (07/03/2007-07/04/2016) facilitado por la CHS.

\section{Escenarios regionalizados de cambio climático en SWAT}

Tras calibrar, validar y analizar la sensibilidad de SWAT, éste puede ser lanzado en escenarios pasados y futuros ya que los valores optimizados en los parámetros de la cuenca no varían con el tiempo (Winchell et al., 2013). En coherencia con los objetivos del estudio, se incorporan en SWAT los escenarios de emisión RCP4.5 y RCP8.5 (periodo 2010-2050). Los modelos de circulación global (GCMs) seleccionados se denominan: bcc-csm1-1-m, MPI.ESM.MR y bcc. csm1.1. Se escogen estos modelos puesto que se trata de los GCMs más próximos al promedio anual de todos los modelos en la DHS (serie 2010-2050). Asimismo, con el fin de adecuar las predicciones de precipitación, temperatura máxima y mínima de los tres GCMs al área de estudio, se acoplan en SWAT las proyecciones de éstos a partir de la regionalización estadística realizada por Petisco de Lara (2008a, 2008b) con el método de análogos (Tabla 2).

A partir de la regionalización por análogos y por regresión lineal, AEMET ha incorporado las series futuras, a nivel diario, de las citadas variables para el periodo 2006-2100, utilizando como referencia la serie 1961-2000, en prácticamente la totalidad de su red de estaciones meteorológicas en el territorio nacional (AEMET, 2017). La red de estaciones de AEMET en la DHS, que incluye proyecciones de cambio climático, consta de 127 estaciones de precipitación y 39 de temperatura, de las cuales se consideraron en el estudio las indicadas en la Figura 1 dada su proximidad con la cuenca. Los ficheros a escala diaria de estas estaciones, para las variables, GCMs, y escenarios mencionados, fueron adaptados al formato requerido por SWAT (.txt). La operación se realizó automáticamente con un bucle ejecutado en el software bash 4.3 en el que se incorporaron como valores ausentes (NA) los del 29 de febrero, caso de los años bisiestos, no incluidos en las proyecciones de AEMET. Asimismo, la tendencia lineal de las series anuales de precipitación y temperatura, resultado del promedio de los tres GCMs utilizados, se evaluó mediante una regresión lineal analizando la significancia de dicha tendencia con el contraste no paramétrico de Mann-Kendall (Kendall, 1976; Sneyers, 1992). 
Tabla 2. Diferencias de cada GCM con el promedio anual del total de modelos en la DHS (periodo 2010-2050) regionalizados con análogos. Elaborado a partir de AEMET (2017).

\begin{tabular}{|c|c|c|c|c|c|c|c|c|c|}
\hline \multicolumn{5}{|c|}{ RCP8.5 } & \multicolumn{5}{|c|}{ RCP4.5 } \\
\hline \multicolumn{2}{|c|}{ Nombre modelo } & \multirow{2}{*}{$\begin{array}{c}\operatorname{Precp}^{\star}(\%) \\
-0.406\end{array}$} & \multirow{2}{*}{$\begin{array}{c}\text { Tmáx }\left({ }^{\circ} \mathbf{C}\right) \\
0.324\end{array}$} & \multirow{2}{*}{$\begin{array}{c}\text { Tmín }\left({ }^{\circ} \mathbf{C}\right) \\
0.212\end{array}$} & \multicolumn{2}{|c|}{ Nombre modelo } & \multirow{2}{*}{$\begin{array}{c}\operatorname{Precp}^{\star}(\%) \\
5.333\end{array}$} & \multirow{2}{*}{$\frac{\text { Tmáx }\left({ }^{\circ} \mathbf{C}\right)}{0.204}$} & \multirow{2}{*}{$\begin{array}{c}\text { Tmín }\left({ }^{\circ} \mathbf{C}\right) \\
0.170\end{array}$} \\
\hline ACCESS1.0 & Australia & & & & ACCESS1.0 & Australia & & & \\
\hline ACCESS 1.3 & Australia & 9.230 & 0.144 & 0.159 & bcc.csm1.1.m & China & 0.313 & 0.078 & 0.073 \\
\hline bcc.csm1.1.m & China & 0.610 & - & - & bcc.csm1.1 & China & 2.496 & 0.044 & 0.027 \\
\hline bcc.csm1.1 & China & -5.823 & 0.110 & 0.025 & BNU.ESM & China & -7.407 & 0.251 & 0.203 \\
\hline BNU.ESM & China & 2.230 & 0.074 & 0.099 & CMCC.CM & Italia & 2.730 & - & - \\
\hline CMCC.CESM & Italia & -4.560 & -0.176 & -0.015 & CMCC.CMS & Italia & -0.767 & 0.281 & 0.273 \\
\hline CMCC.CM & Italia & -3.353 & 0.157 & 0.172 & CNRM.CM5 & Francia & 6.740 & -0.376 & -0.357 \\
\hline CNRM.CM5 & Francia & 2.784 & -0.563 & -0.541 & GFDL.ESM2G & Estados Unidos & -5.090 & - & - \\
\hline GFDL.ESM2G & Estados Unidos & 2.994 & - & - & GFDL.ESM2M & Estados Unidos & -11.634 & - & - \\
\hline HadGEM2.CC & Reino Unido & -4.066 & 0.527 & 0.479 & Inm.cm 4 & Rusia & 2.826 & -0.879 & -0.830 \\
\hline Inm.cm 4 & Rusia & 4.477 & -0.903 & -0.865 & IPSL.CM5A.LR & Francia & -13.434 & - & - \\
\hline IPSL.CM5A.LR & Francia & -1.166 & - & - & IPSL.CM5A.MR & Francia & -5.274 & 0.378 & 0.310 \\
\hline MIROC5 & Japón & 1.904 & 0.207 & 0.202 & IPSL.CM5B.LR & Francia & 10.713 & - & - \\
\hline MIROC.ESM & Japón & -7.506 & 0.640 & 0.555 & MIROC5 & Japón & -2.024 & 0.378 & 0.303 \\
\hline MPI.ESM.LR & Alemania & 4.307 & 0.050 & 0.042 & MIROC.ESM & Japón & -2.387 & 0.544 & 0.507 \\
\hline MPI.ESM.MR & Alemania & -0.026 & -0.003 & 0.005 & MPI.ESM.LR & Alemania & 3.813 & -0.116 & -0.137 \\
\hline \multirow[t]{2}{*}{ MRI.CGCM3 } & Japón & -1.626 & -0.590 & -0.528 & MPI.ESM.MR & Alemania & 4.450 & -0.006 & 0.040 \\
\hline & & & & & MRI.CGCM3 & Japón & 8.600 & -0.782 & -0.583 \\
\hline
\end{tabular}

Nota: en gris se indican los GCMs con menores diferencias en cada variable. ${ }^{*}$ Precp: precipitación.

\section{RESULTADOS Y DISCUSIÓN}

\section{Calibración, validación y análisis de sensibilidad con NSE y KGE}

En la calibración, validación y análisis de sensibilidad de SWAT se contrastaron las mediciones del caudal, a nivel mensual, en la salida de la cuenca con las simulaciones de éste. Los estimadores de la bondad del ajuste, aplicados en la serie de validación y calibración, indican que el caudal simulado adapta su comportamiento a los datos observados. El periodo con caudales medidos (01/01/1996-31/12/2012) es dividido para destinar seis años a validación (01/01/1996-31/12/2001) y 11 años a calibración (01/01/2002-31/12/2012). De este modo, se escoge para calibrar SWAT la serie de mediciones más actual, que presenta menores lagunas y errores de medición. En el caudal simulado en ambas series se destina el primer año como periodo de calentamiento (NYSKIP). Por tanto, este año es eliminado en cada simulación evitando así parcialmente el sesgo inicial del modelo en la simulación (Arnold et al., 2012, Figura 4).

El ajuste con NSE como función objetivo muestra, en calibración, que de las 2000 simulaciones lanzadas (distribución de probabilidad o 95PPU: Abbaspour, 2015) la mejor simulación obtenida con SUFI-2 se adapta satisfactoriamente al caudal observado (Figura 4). Los resultados de la bondad del ajuste mejoran sensiblemente en la serie de calibración con respecto a la de validación, siendo clasificados en ambos casos como buenos y muy buenos según Moriasi et al. (2007). Asimismo, en ambas series SWAT infraestima el caudal observado

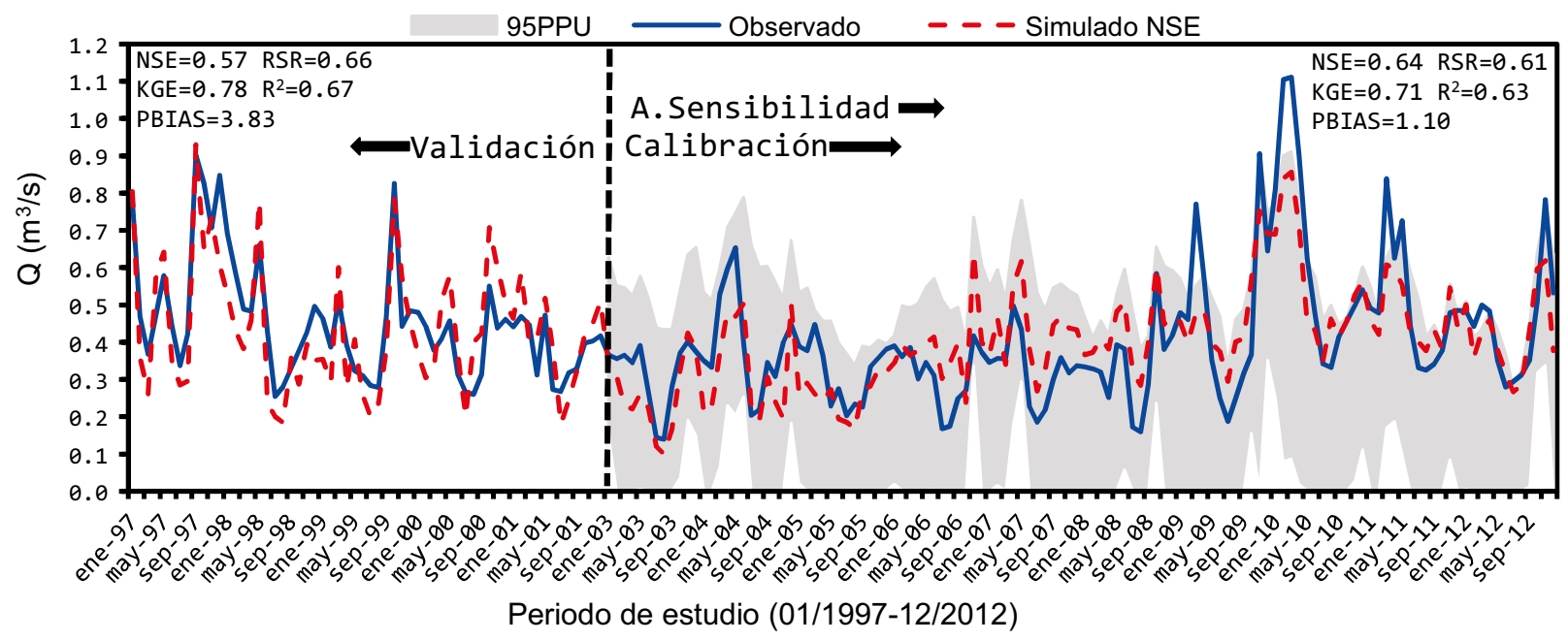

Figura 4. Ajuste de SWAT en validación y calibración con NYSKIP=1 y NSE como función objetivo. 


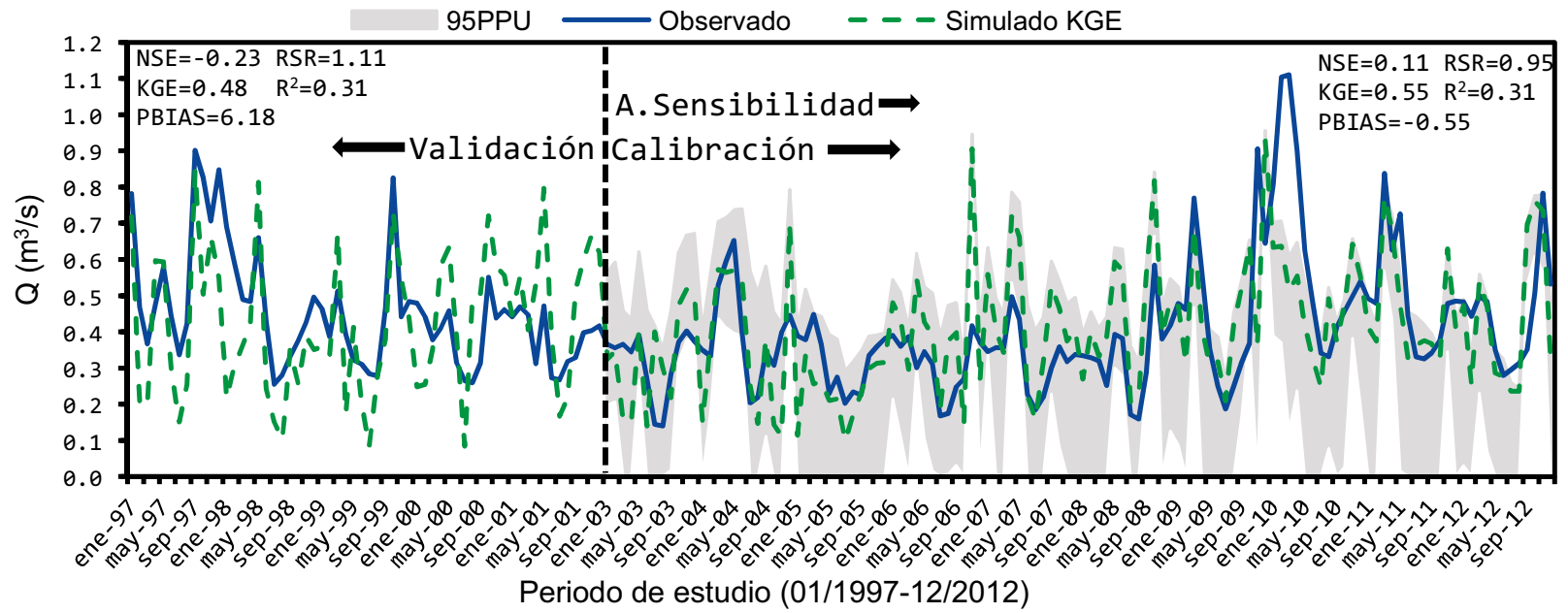

Figura 5. Ajuste de SWAT en validación y calibración con NYSKIP=1 y KGE como función objetivo.

(PBIAS positivo). Sin embargo, el modelo ajusta correctamente el caudal base simulado al observado (ausencia de caudal base nulo), es decir, consigue reproducir los aportes de origen subterráneo recibidos en el embalse del Taibilla durante el periodo de estudio. Además, los caudales punta son simulados de un modo aceptable en dicho periodo pese a la subestimación de los meses 02 y 03 del año 2010. Esta infraestimación indica probablemente, por parte del modelo, una respuesta lenta ante precipitaciones intensas (posible conversión deficitaria lluvia-escorrentía).

La eficiencia de Kling-Gupta (KGE) fue escogida como función objetivo ya que a menudo proporciona un ajuste robusto frente a observaciones con numerosos caudales punta (Figura 5).

Debido a la naturaleza de $K G E$ como función objetivo, el caudal simulado (Figura 5) presenta mayor número de picos (caudales punta) que el simulado con NSE (Figura 4). Esta fluctuación del simulado con $K G E$ provoca que, en la serie de validación, SWAT infraestime el caudal observado de un modo perceptible $(P B I A S=6.18)$, mientras que en calibración lo sobreestima tímidamente $(P B I A S=-0.55)$. Por ello, la representación del caudal base con $K G E$ empeora respecto a la simulación realizada con los parámetros hidrológicos empleando NSE como función objetivo. No obstante, la mencionada fluctuación del caudal simulado favorece que en los meses 02 y 03 del 2010 el simulado se aproxime al observado. Como consecuencia de lo enunciado, en validación y calibración, $K G E$ muestra una bondad del ajuste inferior a la obtenida con NSE (Tabla 3). Estadísticos como NSE, KGE y $R^{2}$ se alejan de rangos satisfactorios según la clasificación de Moriasi et al. (2007) y Gupta et al. (2009).

Tabla 3. Resultados de la bondad del ajuste en validación y calibración con NYSKIP $=1$ en ambas series.

\begin{tabular}{|c|c|c|c|c|c|}
\hline \multicolumn{2}{|c|}{ Estimadores } & \multicolumn{2}{|c|}{$\begin{array}{l}\text { Serie de validación } \\
(01 / 1997-12 / 2001)\end{array}$} & \multicolumn{2}{|c|}{$\begin{array}{c}\text { Serie de calibración } \\
(01 / 2003-12 / 2012)\end{array}$} \\
\hline \multirow[t]{2}{*}{ Nombre } & \multirow[t]{2}{*}{ Óptimo } & \multicolumn{4}{|c|}{ Caudal calibrado } \\
\hline & & $(N S E)$ & $(K G E)$ & (NSE) & $(K G E)$ \\
\hline NSE & 1 & 0.57 & -0.23 & 0.64 & 0.11 \\
\hline$K G E$ & 1 & 0.78 & 0.48 & 0.71 & 0.55 \\
\hline PBIAS & $< \pm 10$ & 3.83 & 6.18 & 1.10 & -0.55 \\
\hline$R S R$ & 0 & 0.66 & 1.11 & 0.61 & 0.95 \\
\hline$R^{2}$ & 1 & 0.67 & 0.31 & 0.63 & 0.31 \\
\hline
\end{tabular}

De este modo, la modelación hidrológica en la cuenca, para el periodo presente y futuro, se realiza a partir de los resultados proporcionados con NSE como función objetivo. Dichos resultados se trasladan a los parámetros hidrológicos a través de la columna valor fijado en la Tabla 4. Se corrigen así los valores originales de los 14 parámetros seleccionados para representar el comportamiento hidrológico de la cuenca alta del río Taibilla (modelización hidrológica inversa). Estos parámetros, junto con sus rangos de valores iniciales en la Iteración uno, fueron escogidos a partir de experiencias de modelización con objetivos similares a los enunciados en este estudio (Abbaspour, 2015; Abbaspour et al., 2015; Gomariz-Castillo, 2015; Senent-Aparicio et al., 2017).

Los parámetros de la Tabla 4 poseen diferencias notables en el análisis de sensibilidad (iteración 5). ALPHA_BF.gw, SOL_K (...). sol y CN2.mgt se muestran significativamente sensibles a $K G E$ como función objetivo ( $P$-valor $\leq 0.05)$. Por ello, un cambio en dichos parámetros afecta en gran medida a los resultados del modelo. El análisis con NSE presenta un número mayor de parámetros con sensibilidad significativa que en el caso anterior $(P$-valor $\leq 0.05)$, favoreciendo así la elección de NSE como función objetivo. ALPHA_BF.gw, CN2.mgt, SOL_BD (...).sol y SOL_AWC(...).sol son los que poseen mayor sensibilidad. Dado que los parámetros ALPHA_BF.gw y CN2.mgt también mostraron sensibilidad significativa con KGE como función objetivo, se considera que ambos resultan esenciales en el comportamiento y dinámica hidrológica de la cuenca alta del Taibilla. Análogamente, los parámetros de las capas de suelo (base de datos .sol) presentan un comportamiento relevante con NSE y KGE como funciones objetivo.

\section{Análisis de las proyecciones de cambio climático}

Los GCMs seleccionados, en la zona de estudio con la regionalización estadística de análogos, se denominan: bcc-csm1-1-m, MPI. ESM.MR y bcc.csm1.1. En la Figura 6 se muestran, para las variables precipitación y temperatura media, los promedios anuales de las simulaciones resultantes de estos GCMs a escala diaria en la cuenca alta del Taibilla. Dichas simulaciones se obtuvieron a nivel de unidad de respuesta hidrológica (HRU) por lo que fueron agregadas espacialmente a escala de cuenca.

Durante las próximas décadas, en los escenarios de emisión RCP4.5 y RCP8.5, el promedio de la precipitación para los GCMs implementados presenta una variabilidad interanual notable (Figura 6). El análisis mediante el contraste no paramétrico de Mann-Kendall (Kendall, 1976; Sneyers, 1992) no detecta tendencias significativas. El escenario RCP8.5 
Tabla 4. Rango de variación de los parámetros hidrológicos en SUFI-2: iteraciones 1 y 5 de 2000 simulaciones con NSE como función objetivo.

\begin{tabular}{|c|c|c|c|}
\hline Parámetro & Descripción (unidad) & $\begin{array}{l}\text { Rango Iter1 } \\
\text { (Mín.....Máx.) }\end{array}$ & $\begin{array}{l}\text { Valor fijado Iter5 } \\
\quad \text { (NSE) }\end{array}$ \\
\hline R_CN2.mgt & $\begin{array}{l}\text { CN2: número de curva de escorrentía inicial, según el SCS (Soil Conservation Service), para la } \\
\text { condición de humedad II (humedad media). Adimensional. }\end{array}$ & {$[-0.2 \ldots 0.2]$} & -0.158 \\
\hline V_ALPHA_BF.gw & $\alpha_{g w}:$ factor alfa del caudal base (1/días). & {$[0.0 \ldots 1.0]$} & 0.001 \\
\hline V_GW_DELAY.gw & $\delta_{g w}:$ periodo de retardo de las aguas subterráneas (días). & {$[30.0 . .450 .0]$} & 110.946 \\
\hline V_GWQMN.gw & $\begin{array}{l}a q_{\text {sththrq }} \text { : umbral de profundidad del agua en el acuífero poco profundo necesario para que se } \\
\text { produzca el flujo de retorno hacia la superficie }\left(\mathrm{mm} \mathrm{H}_{2} \mathrm{O}\right) \text {. }\end{array}$ & {$[0.0 \ldots .2 .0]$} & 0.851 \\
\hline R_SOL_AWC(x).sol & $\mathrm{AWC}_{\mathrm{ly}}$ : capacidad de agua disponible en la capa de suelo $\mathrm{x}\left(\mathrm{mm} \mathrm{H}_{2} \mathrm{O} / \mathrm{mm}\right.$ suelo). & {$[-0.2 \ldots .0 .4]$} & 0.129 \\
\hline R_SOL_K(x).sol & Ksat: conductividad hidráulica saturada de la capa de suelo $\mathrm{x}(\mathrm{mm} / \mathrm{hr})$. & {$[-0.8 \ldots .0 .8]$} & -0.267 \\
\hline R_SOL_BD(x).sol & $p b$ : Densidad aparente húmeda en la capa de suelo $\mathrm{x}\left(\mathrm{Mg} / \mathrm{m}^{3}\right)$ & {$[-0.5 \ldots .0 .6]$} & 0.784 \\
\hline R_ESCO.hru & ESCO: factor de compensación de la evaporación del suelo (adimensional). & {$[0.8 \ldots .1 .0]$} & 0.792 \\
\hline V_GW_REVAP.gw & $\beta_{r e v}:$ coeficiente "Revap" de las aguas subterráneas (adimensional). & {$[0.0 \ldots . .0 .2]$} & 0.279 \\
\hline R_SLSUBBSN.hru & $\mathrm{L}_{\mathrm{sp}}:$ longitud media de la pendiente $(\mathrm{m})$ & {$[0.0 \ldots . .0 .2]$} & 0.125 \\
\hline V_SFTMP.bsn & $\begin{array}{l}\mathrm{T}_{s-\mathrm{r}} \text { : temperatura de las nevadas. Es la temperatura media del aire en la que la probabilidad de } \\
\text { que la precipitación caiga como nieve es análoga a que caiga como lluvia helada }\left({ }^{\circ} \mathrm{C}\right) .\end{array}$ & {$[-5.0 \ldots .5 .0]$} & -3.716 \\
\hline V_REVAPMN.gw & $\begin{array}{l}a q_{\text {sththrrvp: }} \text { umbral de profundidad del agua en el acuífero poco profundo necesario para que } \\
\text { se produzca el coeficiente "Revap" o la percolación del agua hacia el acuífero profundo (mm } \\
\mathrm{H}_{2} \mathrm{O} \text { ). }\end{array}$ & {$[0.0 \ldots 10.0]$} & 5.576 \\
\hline R_HRU_SLP.hru & slp: inclinación media de la pendiente $(\mathrm{m} / \mathrm{m})$ & {$[0.0 \ldots . .0 .2]$} & 0.136 \\
\hline R_OV_N.hru & n: valor del coeficiente de rugosidad "n" de Manning para el flujo superficial (adimensional). & {$[-0.2 \ldots .0 .0]$} & -0.067 \\
\hline
\end{tabular}

presenta una tendencia moderadamente decreciente con una reducción de la precipitación media de $8.4 \mathrm{~mm} /$ década, mientras que el escenario RCP4.5 muestra una tendencia prácticamente constante $(+0.3 \mathrm{~mm} /$ década). El promedio de la temperatura en cambio registra un claro incremento en el periodo de estudio (2010-2050). Los dos escenarios considerados prevén un aumento de la temperatura estadísticamente significativo (P-valor<0.001) en dicho periodo (Figura 6). El incremento previsto es de 0.31 y $0.25^{\circ} \mathrm{C} /$ década para los escenarios RCP8.5 y RCP4.5 respectivamente. Estas modificaciones resultan coherentes con los cambios proyectados, a lo largo del siglo XXI, en CEDEX (2012) y Senent-Aparicio et al. (2017) en la cabecera de la Demarcación Hidrográfica del río Segura (DHS). Del mismo modo, las tendencias identificadas (Figura 6) concuerdan con las variaciones, para precipitación y temperatura, estimadas a escala global en Allen et al. (2000) e IPCC (2013) debido al aumento de las emisiones y concentración atmosférica de gases de efecto invernadero (GEI).

Así mismo, la evolución de los diferentes modelos, en las variables y escenarios de emisión analizados (Figura 6), indica que el que el GCM bcc.csm1.1 muestra mayor variabilidad interanual (mayor número de picos) que los restantes con respecto a la serie promedio. A pesar de ello, las últimas décadas del periodo de estudio, especialmente la 2040-2050, presentan una convergencia y estabilización de las proyecciones de los modelos utilizados, aspecto ya señalado en IPCC (2013). No obstante, este informe señala que las predicciones de temperaturas y precipitaciones futuras poseen una gran incertidumbre asociada al GCM utilizado. La variable precipitación muestra, además, elevada incertidumbre en función de la técnica de regionalización implementada (IPCC, 2013).

En virtud de las proyecciones de cambio climático analizadas, para las variables precipitación y temperatura, se estima que el clima actual de la cuenca alta del Taibilla, clasificado en Boix-Fayos et al. (2007) como mediterráneo subhúmedo en transición al semiárido, derivará hacia una climatología de tipo semiárida-árida. Dichos cambios se deben principalmente a los incrementos, con tendencia significativa (P-valor $<0.001)$, identificados en la temperatura (Figura 6).

\section{Respuesta hidrológica en los periodos de estudio}

En este apartado, se analiza la respuesta hidrológica de la cuenca en el periodo actual (2000-2010) junto con el corto plazo (2021-2030) y el mediano plazo (2041-2050) como consecuencia de las proyecciones de cambio climático acopladas al modelo SWAT tras evaluar la bondad del mismo (principalmente mediante el fichero Output.std obtenido en cada simulación). Los promedios anuales de los elementos del balance hídrico mostrados (Tabla 5) proceden de la agregación temporal y espacial realizada, a escala de cuenca, con el software SWAT-CHECK (White et al., 2012) durante los periodos mencionados. En la Tabla 5 se incluyen, para el corto y el mediano plazo, los resultados procedentes del promedio de los tres GCMs considerados en el estudio. Los valores medios de precipitación, escorrentía superficial y recarga de acuíferos se relacionan en mm/año, mientras que la temperatura media en ${ }^{\circ} \mathrm{C}$. Así mismo, se indican las modificaciones que dichas variables presentan en los periodos y escenarios futuros con respecto a sus respectivos valores en el periodo presente (2000-2010).

Los resultados obtenidos (Tabla 5) aportan valores y tendencias verosímiles en la cuenca alta del Taibilla dada la climatología actual y futura de la misma. Así mismo, los cambios registrados en las variables del balance hídrico (Tabla 5) concuerdan con las modificaciones mostradas en CEDEX (2012), CHS (2015a) y Senent-Aparicio et al. (2017) para la cabecera de la DHS durante el periodo 2000-2050. Ello indica, junto con los resultados de la calibración y validación del modelo, una correcta elección de la función objetivo, la eficiencia de NSE, así como de los 14 parámetros hidrológicos seleccionados para representar el comportamiento hídrico de la cuenca.

La precipitación media anual en la cuenca alcanza, actualmente, los $585 \mathrm{~mm}$ según los registros facilitados por AEMET mientras que SWAT estima en el periodo 2000-2010 596.46 mm/año (Tabla 5) debido a que el modelo completa los valores ausentes existentes en las series de AEMET. Los periodos 2021-2030 y 2041-2050, para el escenario RCP4.5, muestran reducciones de la pluviometría media de en torno al $3.8 \%$ con respecto a los $596.46 \mathrm{~mm} /$ año del 2000-2010. En cambio, el descenso del escenario RCP8.5, en ambos periodos, se sitúa en el $12.7 \%$. 

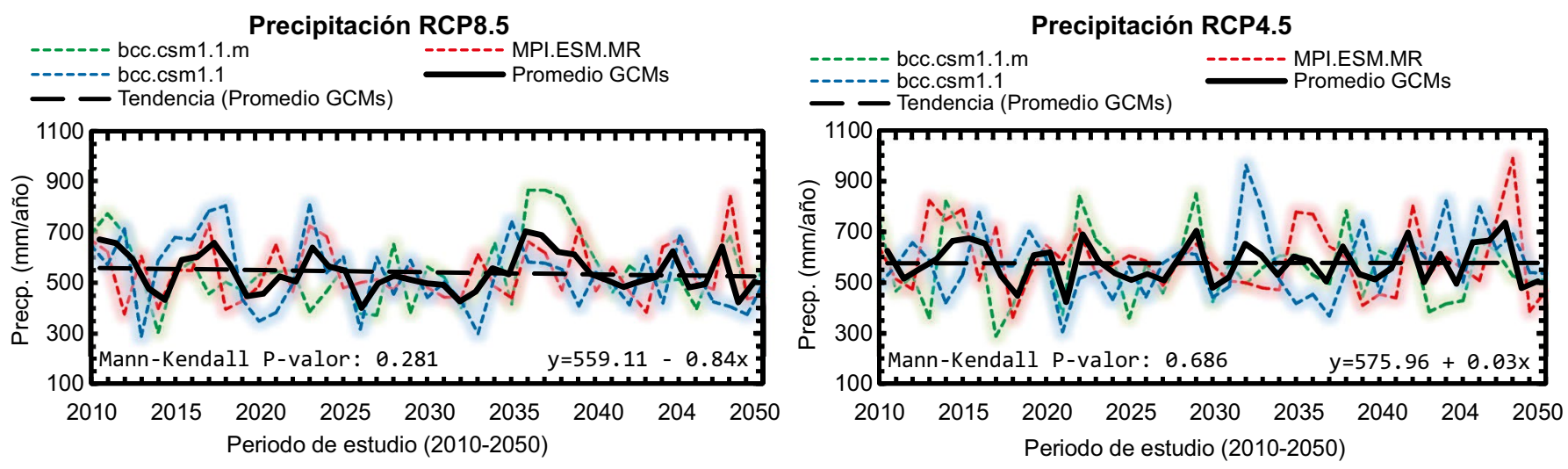

Temperatura RCP8.5
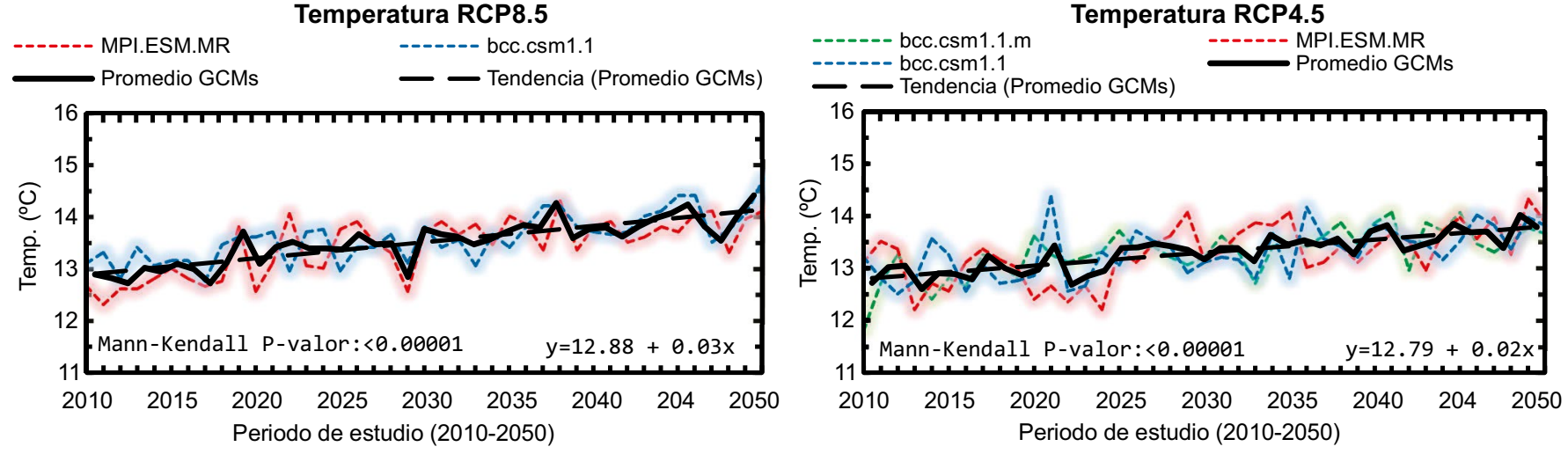

Figura 6. Evolución de las proyecciones de cambio climático en la cuenca (periodo 2010-2050).

A pesar de ello, en dichos escenarios, las modificaciones estimadas en la precipitación para el promedio del periodo 2010-2050 mostraron tendencias no significativas.

A diferencia de la pluviometría, la temperatura media en la cuenca registra para las próximas décadas incrementos significativos $(\mathrm{P}$-valor $<0.001)$ en los escenarios de emisión considerados. En el RCP4.5, con respecto al periodo actual, la temperatura aumentará $1.47^{\circ} \mathrm{C}$ en el corto y el mediano plazo, mientras que en el RCP8.5 ascenderá $1.72^{\circ} \mathrm{C}$ (Tabla 5). Dichos aumentos se aproximan al umbral de los $2{ }^{\circ} \mathrm{C}$ fijados como punto de no retorno a partir del cual la influencia del cambio climático sobre los recursos hídricos y ecosistemas asociados tendrá efectos irreversibles (Green et al., 2011; IPCC, 2013; Klove et al., 2014). Así mismo, los citados cambios afectarán a las tasas de evapotranspiración real (ETR) y potencial (ETP) estimadas en el periodo presente con SWAT en $402.33 \mathrm{~mm} /$ año y $947.97 \mathrm{~mm} /$ año respectivamente. En coherencia con los resultados de CEDEX (2012) para el sureste mediterráneo, durante las próximas décadas, se prevén en la cuenca reducciones de la ETR, debido a la menor disponibilidad de agua de lluvia, e incrementos en la ETP por el aumento previsto de la temperatura media.

Las modificaciones estimadas para precipitación y temperatura provocarán una disminución de los recursos hídricos superficiales y subterráneos en la cuenca, cuya disponibilidad neta se verá además condicionada por las elevadas tasas de ETR propias del sureste mediterráneo. El 64\% de la lluvia caída genera escorrentía superficial (número de curva promedio de 64.06) debido a la humedad, tipos y usos del suelo (zona próxima al régimen natural). En el periodo 2000 2010, la escorrentía superficial se sitúa en los $40.04 \mathrm{~mm} / \mathrm{año}$ (Tabla 5). En el escenario RCP4.5 para el corto y el mediano plazo desciende un $10.2 \%$, mientras que en el RCP8.5 disminuye un 18.6\%.

La recarga de acuíferos presenta en el periodo actual un valor promedio de $103.01 \mathrm{~mm} /$ año (Tabla 5). En el escenario RCP4.5, durante las series 2021-2030 y 2041-2050, se reduce un $4.4 \%$ y en el RCP8.5 un 16.3\%. Estos cambios provocarán reducciones en las tasas de retorno de acuíferos y por ende del caudal base o ecológico de los cursos superficiales afectando negativamente a los ecosistemas

Tabla 5. Respuesta hidrológica de la cuenca alta del Taibilla en los periodos de estudio.

\begin{tabular}{|c|c|c|c|c|c|}
\hline \multirow{2}{*}{ Variable } & \multirow{2}{*}{ Periodo 2000-2010 } & \multicolumn{2}{|c|}{ Periodo 2021-2030 } & \multicolumn{2}{|c|}{ Periodo 2041-2050 } \\
\hline & & RCP4.5 & RCP8.5 & RCP4.5 & RCP8.5 \\
\hline Precipitación & 596.46 & $556.78(-6.65 \%)$ & $521.62(-12.55 \%)$ & $590.69(-0.97 \%)$ & $519.32(-12.93 \%)$ \\
\hline Temperatura & 11.97 & $13.21\left(+1.24^{\circ} \mathrm{C}\right)$ & $13.44\left(+1.47^{\circ} \mathrm{C}\right)$ & $13.66\left(+1.69^{\circ} \mathrm{C}\right)$ & $13.93\left(+1.96^{\circ} \mathrm{C}\right)$ \\
\hline Escorrentía superficial & 40.04 & $34.89(-12.87 \%)$ & $32.69(-18.37 \%)$ & $37.01(-7.56 \%)$ & $32.54(-18.73 \%)$ \\
\hline Percolación/recarga acuíferos & 103.01 & $92.72(-9.99 \%)$ & $89.34(-13.27 \%)$ & $104.14(+1.10 \%)$ & $83.07(-19.36 \%)$ \\
\hline
\end{tabular}


asociados (Klove et al., 2014). No obstante, los descensos estimados en la recarga de acuíferos resultan menores que los obtenidos para la escorrentía superficial durante las próximas décadas. Además, los elevados tiempos de tránsito y residencia del flujo hídrico en el subsuelo, variables desde días a miles de años, impiden identificar de un modo directo los efectos del cambio climático sobre las aguas subterráneas (Green et al., 2011). De hecho, en la cuenca, el escenario RCP4.5 prevé un incremento del $1.1 \%$ en las tasas de recarga durante el periodo 2041-2050. Del mismo modo, determinados estudios realizados en la DHS proyectan aumentos puntuales de la recarga de acuíferos durante los próximos decenios (Pulido-Velázquez et al., 2015). Ello indica que actualmente existe una gran incertidumbre asociada a los escenarios de emisión, GCMs y técnicas de regionalización estadística empleadas en las proyecciones de cambio climático.

\section{CONCLUSIONES}

En el presente estudio, durante la calibración y validación de SWAT en la cuenca alta del río Taibilla, se escogió la eficiencia de NSE como función objetivo debido a la mejora que éste aporta, con respecto a la eficiencia de $K G E$, en los resultados de la bondad del ajuste entre caudales observados y simulados. De este modo, mediante NSE, SWAT simula adecuadamente el hidrograma observado en la salida de la cuenca para la serie 1996-2012.

Así mismo, las proyecciones de cambio climático acopladas a SWAT considerando el forzamiento radiativo aportado por los escenarios de emisión RCP4.5 y RCP8.5 para los GCMs bcc-csm1-1-m, MPI. ESM.MR y bcc.csm1.1, con la regionalización estadística de análogos, muestran que en el periodo 2010-2050 la precipitación media en la cuenca posee una elevada variabilidad interanual con tendencias no significativas en ambos escenarios. El escenario RCP8.5 prevé reducciones de $8.4 \mathrm{~mm} /$ década y el RCP4.5 un moderado incremento de $0.3 \mathrm{~mm} /$ década. Sin embargo, la temperatura media presenta un claro aumento con tendencias significativas $(\mathrm{P}$-valor $<0.001)$ en los dos escenarios. El incremento previsto es de $0.31^{\circ} \mathrm{C} /$ década en el escenario RCP8.5 y de $0.25^{\circ} \mathrm{C} /$ década en el RCP4.5.

En cuanto al análisis de la respuesta hidrológica ante las proyecciones de cambio climático consideradas, los resultados indican que la cuenca experimentará en las series simuladas (2021-2030 y 20412050), con respecto al periodo actual (2000-2010), una reducción de la precipitación media de aproximadamente un $4 \%$ en el escenario RCP4.5 y de un 13\% en el RCP8.5. La temperatura se incrementará $1.5^{\circ} \mathrm{C}$ y $1.7^{\circ} \mathrm{C}$ en ambos escenarios respectivamente. Por su parte, la escorrentía superficial disminuirá un $10 \%$ en el RCP4.5 y un $19 \%$ en el RCP8.5. No obstante, la recarga de acuíferos presenta menores reducciones ante las citadas predicciones de cambio climático que la escorrentía superficial, aspecto ya señalado en determinados estudios realizados en la DHS. En el escenario RCP4.5 se prevé una disminución de la recarga del $4.5 \%$ y en el RCP8.5 del 16\%. Dichas modificaciones en las principales variables del balance hídrico resultan coherentes con la disminución de las aportaciones naturales de referencia, prevista en un $11 \%$, para la DHS durante el siglo XXI (CEDEX, 2012; SenentAlonso y García-Aróstegui, 2014). Se estima que la verosimilitud de los resultados obtenidos radica principalmente en la elección de los tres modelos de cambio climático que presentan menores diferencias con respecto al total de GCMs disponibles en la DHS para el periodo 2006-2100. A partir de las alteraciones enunciadas se prevé que el clima actual de la cuenca, mediterráneo subhúmedo en transición al semiárido, derive hacia una climatología de tipo semiárida-árida.

La MCT abastece a numerosos municipios de las provincias de Murcia (44), Alicante (35) y Albacete (dos). Las fuentes de suministro que utiliza proceden del trasvase Tajo-Segura y extraordinarios (57.4\%), de la desalación de agua marina (3.4\%) y de los aportes almacenados en el embalse del Taibilla (39.2\%). Aunque estos porcentajes varían en función del año hidrológico considerado, las reducciones obtenidas en las reservas de la cuenca durante las próximas décadas repercutirán negativamente en el suministro de sus demandas urbanas y agrícolas. Por ello, los políticos y gestores del recurso agua deberán establecer estrategias de gestión más eficientes e incrementar los recursos procedentes del trasvase Tajo-Segura y de la desalación de agua marina. No obstante, dicho escenario parece poco probable dada la grave situación de escasez y sequía que atraviesan numerosas demarcaciones hidrográficas españolas durante los últimos años hidrológicos. A su vez, los elevados costes de la desalación provocan que ésta no resulte actualmente una fuente de suministro factible para la agricultura.

\section{AGRADECIMIENTOS}

El presente trabajo se enmarca parcialmente en el proyecto 19325/ PI/15Ref concedido por la Fundación Séneca-Agencia de la Ciencia y la Tecnología (Región de Murcia, España). Adicionalmente, el primer autor agradece la financiación recibida del Programa Nacional de Formación de Profesorado Universitario (FPU) concedida por el Ministerio Español de Educación, Cultura y Deporte (MECD). Del mismo modo, los autores agradecen las sugerencias realizadas por los revisores anónimos del artículo, cuyos comentarios contribuyeron a mejorar el manuscrito.

\section{REFERENCIAS}

Abbaspour, K.C., 2015, SWAT-Calibration and uncertainty programs (CUP)User Manual: Duebendorf, Swiss Federal Institute of Aquatic Science and Technology (Eawag), 100 pp.

Abbaspour, K.C., Johnson, A., Van Genuchten, M.T., 2004, Estimating uncertain flow and transport parameters using a sequential uncertainty fitting procedure: Vadose Zone Journal, 3(4), 1340-1352.

Abbaspour, K.C., Rouholahnejad, E., Vaghefi, S., Srinivasan, R., Yang, H., Klove, B., 2015, A continental-scale hydrology and water quality model for Europe: Calibration and uncertainty of a high-resolution large-scale SWAT model: Journal of Hydrology, 524, 733-752.

Abbaspour, K.C., Yang, J., Maximov, I., Siber, R., Bogner, K., Mieleitner, J., Zobrist, J., Srinivasan, R., 2007, Modelling hydrology and water quality in the pre-alpine/alpine Thur watershed using SWAT: Journal of Hydrology, 333(2), 413-430.

AEMET (Agencia Estatal de Meteorología), 2017, Servicios climáticos. Proyecciones climáticas para el siglo XXI: <http://www.aemet.es/es/ serviciosclimaticos/cambio_climat>, consulta 01/2017.

Aguilar-García, R., Ortega-Guerrero, M.A., 2017, Análisis de la dinámica del agua en la zona no saturada en un suelo sujeto a prácticas de conservación: implicaciones en la gestión de acuíferos y adaptación al cambio climático: Revista Mexicana de Ciencias Geológicas, 34(2), 91-104.

Al Kuisi, M., El-Naqa., A., 2013, GIS based Spatial Groundwater Recharge estimation in the Jafr basin, Jordan-Application of WetSpass models for arid regions: Revista Mexicana de Ciencias Geológicas, 30(1), 96-109.

Allen, M.R., Stott, P.A., Mitchell, J.F.B., Schnur, R., Delworth, T.L., 2000, Quantifying the uncertainty in forecasts of anthropogenic climate change: Nature, 407, 617-620.

Arnold, J.G., Kiniry, J.R., Srinivasan, R., Williams, J.R., Haney, E.B., Neitsch, S.L., 2012, Input/output documentation version 2012: Texas, Texas Water Resources Institute, $650 \mathrm{pp}$.

Arnold, J.G., Srinivasan, R., Muttiah, R.S., Williams, J.R., 1998, Large area hydrologic modeling and assessment part I: model development: Journal of the American Water Resources Association, 34(1), 73-89.

AYESA (AYESA Ingeniería), 2010a, Valoración del Estado Ambiental del río Taibilla. T.M. Nerpio (Albacete): Murcia, Confederación Hidrográfica 
del Segura, $59 \mathrm{pp}$.

AYESA (AYESA Ingeniería), 2010b, Apéndice 1. Resultados del modelo SIMPA de la cuenca del río Taibilla. Valoración del Estado Ambiental del río Taibilla. T.M. Nerpio (Albacete): Murcia, Confederación Hidrográfica del Segura, 55 pp.

Beven, K., Binley, A., 1992, The Future of Distributed Models: Model Calibration and Uncertainty Prediction: Hydrological Processes, 6(3), 279-298.

Bindoff, N.L., Stott, P.A., AchutaRao, K.M., Allen, M.R., Gillett, N., Gutzler, D., Hansingo, K., Hegerl, G., Hu, Y., Jain, S., Mokhov, I.I., Overland, J., Perlwitz, J., Sebbari, R., Zhang, X., 2013, Detection and Attribution of Climate Change: from Global to Regional, en Climate Change 2013: The Physical Science Basis. Contribution of Working Group I to the Fifth Assessment Report of the Intergovernmental Panel on Climate Change: Cambridge, United Kingdom, and New York, United States of America, Cambridge University Press, 86 pp.

Bobal, P., Sir, B., Richnavsky, J., Unucka, J., 2010, Analysis of the impact of land cover spatial structure change on the erosion processes in the catchment: Acta Montanistica Slovaca, 15(4), 269-276.

Boix-Fayos, C., Barberá, G.G., López-Bermúdez, F., Castillo, V.M., 2007, Effects of check dams, reforestation and land-use changes on river channel morphology: Case study of the Rogativa catchment (Murcia, Spain): Geomorphology, 91, 103-123.

Boix-Fayos, C., De Vente, J., Martínez-Mena, M., Barberá, G.G., Castillo, V.M., 2008, The impact of land use change and check-dams on catchment sediment yield: Hydrological Processes, 22(25), 4922-4935.

Cabezas, F., 2015, Análisis Estructural de Modelos Hidrológicos y de Sistemas de Recursos Hídricos en Zonas Semiáridas: Murcia, Universidad de Murcia, tesis doctoral, $290 \mathrm{pp}$.

CEDEX (Centro de Estudios y Experimentación de Obras Públicas), 2012, Estudio de los impactos del cambio climático en los recursos hídricos y las Masas de Agua. Efecto del cambio climático en los recursos hídricos disponibles en los sistemas de explotación: Madrid, informe técnico final, $296 \mathrm{pp}$

CHS (Confederación Hidrográfica del Segura), 2015a, Plan Hidrológico de la Demarcación del Segura 2015/21. Memoria: Murcia, 806 pp.

CHS (Confederación Hidrográfica del Segura), 2015b, Trabajos de Medida de Caudales en Manantiales y Niveles Hidrométricos y Piezométricos en Humedales de la Cuenca del Segura. Memoria: Murcia, $121 \mathrm{pp}$.

CHS (Confederación Hidrográfica del Segura), 2015c, Anexo 2. Inventario de Recursos Hídricos: Murcia, 152 pp.

CHS (Confederación Hidrográfica del Segura), 2015d, Anexo I, Anejo 3. Fichas de Caracterización de las unidades de Demanda Urbana: Murcia, 46 pp.

CHS (Confederación Hidrográfica del Segura), 2015e, Anexo V, Anejo 3. Fichas de Caracterización de las Unidades de Demanda Agraria: Murcia, 272 pp.

CHS (Confederación Hidrográfica del Segura), 2015f, Anexo I, Anejo 4: Identificación de Masas de Agua relacionadas con Red Natura 2000. Inventario de Hábitats y Especies relacionadas con el Medio Acuático: Murcia, $95 \mathrm{pp}$.

CHS (Confederación Hidrográfica del Segura), 2015g, Anexo II, Anejo 4: Relación entre las Zonas Protegidas y las Masas de Agua de la Demarcación: Murcia, $44 \mathrm{pp}$.

CHS (Confederación Hidrográfica del Segura), 2015h, Anexo II, Anejo 6: Descripción detallada del Modelo de Simulación de la cuenca del Segura: Murcia, 116 pp.

CHS (Confederación Hidrográfica del Segura), 2015i, Anejo 12. Caracterización adicional de las masas de agua subterránea en riesgo de no cumplir los objetivos medioambientales en 2015. MASub 070.032 Caravaca: Murcia, $62 \mathrm{pp}$.

CHS (Confederación Hidrográfica del Segura), 2017, Cartografía: <https://www. chsegura.es/chs/cuenca/resumendedatosbasicos/cartografia/descargas/>, consulta 08/2017.

CNIG (Centro Nacional de Información Geográfica), 2017: Madrid, <http:// centrodedescargas.cnig.es/CentroDescargas/buscadorCatalogo. do? codFamilia $=02107>$, consulta $04 / 2017$.

Custodio, E., Andreu-Rodes, J.M., Aragón, R., Estrela, T., Ferrer, J., GarcíaAróstegui, J.L., Manzano, M., Rodríguez-Hernández, L., Sahuquillo, A., del Villar, A., 2016, Groundwater intensive use and mining in south-eastern peninsular Spain: Hydrogeological, economic and social aspects: Science of the Total Environment, 559(1), 302-316.
Cynthia, R., Strzepek, K.M., Major, D.C., Iglesias, A., Yates, D.N., McCluskey, A., Hillel, D., 2004, Water resources for agriculture in a changing climate: international case studies: Global Environmental Change, 14, 345-360.

DMA, 2000, Directiva 2000/60/CE del Parlamento Europeo y del Consejo de 23 de octubre de 2000 por la que se establece un marco comunitario de actuación en el ámbito de la política de aguas: Diario Oficial de las Comunidades Europeas, L327, 22.12.2000, 1-73.

DOCE, 1992, Directiva 92/43/CEE del Consejo de 21 de mayo de 1992 relativa a la conservación de los hábitats naturales y de la fauna y flora silvestres, $44 \mathrm{pp}$.

El-Sadek, A., Irvem, A., 2014, Evaluating the impact of land use uncertainty on the simulated streamflow and sediment yield of the Seyhan River basin using the SWAT model: Turkish Journal of Agriculture and Forestry, 38(4), 515-530.

FAO (Food and Agriculture Organization of United Nations), 1990, Guidelines for soil profile description. 3rd Edition: Rome, $70 \mathrm{pp}$.

Gassman, P.W., Reyes, M.R., Green, C.H., Arnold, J.G., 2007, The Soil and Water Assessment Tool: Historical Development, Applications and Future Research Directions: Transactions of the American Society of Agricultural and Biological Engineers (ASABE), 50(4), 1211-1250.

Gassman, P.W., Sadeghi, A.M., Srinivasan, R., 2014, Applications of the SWAT model special section: Overview and insights: Journal of Environmental Quality, 43, 1-8.

Gomariz-Castillo, F.., 2015, Estimación de Variables y Parámetros Hidrológicos y Análisis de su Influencia sobre la Modelización Hidrológica: Aplicación a los Modelos Témez y Swat: Murcia, Universidad de Murcia, tesis doctoral, $861 \mathrm{pp}$, http://hdl.handle.net/10201/50539.

Green, T.R., Taniguchi, M., Kooi, H., Gurdak, J.J., Allen, D.M., Hiscock, K.M., Treidel, H., Aureli, A., 2011, Beneath the surface of global change: Impacts of climate change on groundwater: Journal of Hydrology, 405(3), 532-560.

Guerrero, J.J., Ortiz, A.S., Rubio, R.M., Calle, J.A., Garre, M., 2011, Los Noctuoidea de la Sierra del Taibilla y de la Reserva Natural de la Sierra de las Cabras (Albacete-Murcia, España): Revista de Lepidopterología, 39(155), 285-300.

Gupta, H.V., Kling, H., Yilmaz, K.K., Martínez, G.F., 2009, Decomposition of the mean squared error and NSE performance criteria: Implications for improving hydrological modelling: Journal of SHydrology, 377(1-2), 80-91.

Hargreaves, G.L., Hargreaves, G.H., Riley, J.P., 1985, Agricultural benefits for Senegal River Basin: Journal of Irrigation and Drainage Engineering, 111(2), 113-124.

HWSD, 2012, Harmonized World Soil Database (versión 1.2): Rome, Italy, and Laxenburg, Austria, Food and Agriculture Organization of the United Nations (FAO) and International Institute for Applied Systems Analysis (IIASA), $50 \mathrm{pp}$

HWSD, 2017, Harmonized World Soil Database v. 1.2: Rome, Italy, and Laxenburg, Austria, Food and Agriculture Organization of the United Nations (FAO) and International Institute for Applied Systems Analysis (IIASA), <http://webarchive.iiasa.ac.at/Research/LUC/External-Worldsoil-database/HTML>, consulta: 04/2017.

IGME (Instituto Geológico y Minero de España), 2003, Hojas 0888, Yetas de Abajo; 0889, Moratalla; 0909, Nerpio y 0910 Caravaca de la Cruz; 2a Serie del Mapa Geológico de España,escala 1:50000 (MAGNA50): Madrid, Memorias asociadas al MAGNA de estas hojas: 70 pp, 55 pp, 37 pp, y 35 pp.

IPCC (Intergovernmental Panel on Climate Change), 2013, Climate Change 2013: The Physical Science Basis. Contribution of Working Group I to the Fifth Assessment Report of the Intergovernmental Panel on Climate Change: Cambridge, Reino Unido y New York, Estados Unidos de América, Cambridge University Press, 1552 pp.

Kendall, S., 1976, Time Series, 2nd edition: New York, Oxford University Press, 198 pp.

Klove, B., Ala-Aho, P., Bertrand, G., Gurdak, J.J., Kupfersberger, H., Kvaerner, J., Muotka, T., Mykrä, H., Preda, E., Rossi, P., Bertacchi-Uvo, C., Velasco, E., Pulido-Velázquez, M., 2014, Climate change impacts on groundwater and dependent ecosystems: Journal of Hydrology, 518, 250-266.

Krause, P., Boyle, D.P., Bäse, F., 2005, Comparison of different efficiency criteria for hydrological model assessment: Advances in Geosciences, 5, 89-97.

Ma, L., Ascough II, J.C., Ahuja, L.R., Shaffer, M.J., Hanson, J.D., Rojas, K.W., 2000, Root zone water quality model sensitivity analysis using Monte Carlo simulation: Transactions of the Asabe, 43(4), 883-895. 
MCT (Mancomunidad de los Canales del Taibilla), 2014, Gestión del Servicio, Año 2013: Cartagena, Murcia, reporte, 48 pp.

Melgarejo, J., Molina, A., 2017, La Mancomunidad de los Canales del Taibilla en la provincia de Alicante: Alicante, Mancomunidad de los Canales del Taibilla, Universidad de Alicante, $288 \mathrm{pp}$.

Molina, A., Melgarejo, J., 2015, Water policy in Spain: seeking a balance between transfers, desalination and wastewater reuse: International Journal of Water Resources Development, 32(5), 781-798.

Molina, J.L., Pulido-Velázquez, D., García-Aróstegui, J.L., Pulido-Velázquez, M., 2013, Dynamic Bayesian Networks as a Decision Support tool for assessing Climate Change impacts on highly stressed groundwater systems: Journal of Hydrology, 479, 113-129.

Moriasi, D.N., Arnold, J.G., Van Liew, M.W., Bingner, R.L., Harmel, R.D., Veith, T.L., 2007, Model evaluation guidelines for systematic quantification of accuracy in watershed simulations: Transactions of the Asabe, 50(3), 885-900.

Moutahir, H., Bellot, P., Monjo, R., Bellot-Abad, J.F., Garcia, M., Touhami, I., 2017, Likely effects of climate change on groundwater availability in a Mediterranean region of Southeastern Spain: Hydrological Processes, 31(1), 161-176.

Nash, J.E., Sutcliffe, J.V., 1970, River Flow Forecasting through Conceptual Models part I. A Discussion of Principles: Journal of Hydrology, 10(3), 282-290.

Neitsch, S.L., Arnold, J.G., Kiniry, J.R., Williams, J.R., 2011, Soil and Water Assessment Tool. Theorical Documentation. Version 2009: Texas, Texas Water Resources Institute, $647 \mathrm{pp}$

Pérez-González, M.L., Capra-Pedol, L., Dávila-Hernández, N., Borselli, L., SolísValdez, S., Ortiz-Rodríguez, A.J., 2017, Spatio-temporal land-use changes in the Colima-Villa de Álvarez metropolitan area, and their relationship to floodings: Revista Mexicana de Ciencias Geológicas, 34(2), 78-90.

Petisco de Lara, S.E., 2008a, Método de Regionalización de Precipitación basado en Análogos. Explicación y Validación: Madrid, Agencia Estatal de Meteorología, $36 \mathrm{pp}$.

Petisco de Lara, S.E., 2008b, Método de Regionalización de Temperaturas basado en Análogos. Explicación y Validación: Madrid, Agencia Estatal de Meteorología, 48 pp.

Praskievicz, S., Chang, H., 2009, A review of hydrological modelling of basinscale climate change and urban development impacts: Progress in Physical Geography, 33(5), 650-671.

Pulido-Velázquez, D., García-Aróstegui, J.L., Molina, J.L., Pulido-Velázquez, M., 2015, Assessment of future groundwater recharge in semi-arid regions under climate change scenarios (Serral-Salinas aquifer, SE Spain). Could increased rainfall variability increase the recharge rate?: Hydrological Processes, 29(6), 828-844.

Quiñonero-Rubio, J.M., Boix-Fayos, C., De-Vente, J., 2013, Desarrollo y Aplicación de un Índice Multifactorial de Conectividad de Sedimentos a escala de Cuenca: Cuadernos de Investigación Geográfica, 39(2), 203-223.
R Core Team, 2013, R: a language and environment for statistical computing. R-Foundation for Statistical Computing: Vienna Austria, <http://www.Rproject.org/>, consulta: agosto de 2017.

Sánchez-Galiano, J.C., Martí-Ciriquián, P., Fernández-Aracil, P., 2017, Temporary population estimates of mass tourism destinations: The case of Benidorm: Tourism Management, 62, 234-240.

Senent-Alonso, M., García-Aróstegui, J.L., 2014, Sobreexplotación de acuíferos en la cuenca del Segura. Evaluación y perspectivas: Murcia, Fundación Instituto Euromediterráneo del Agua, $231 \mathrm{pp}$.

Senent-Aparicio, J., Pérez-Sánchez, J., Carrillo-García, J., Soto, J., 2017, Using SWAT and Fuzzy TOPSIS to Assess the Impact of Climate Change in the Headwaters of the Segura River Basin (SE, Spain): Water, 9(149), 1-14.

Sneyers, R., 1992, On the use of statistical analysis for the objective determination of climatic change: Meteorologische Zeitschrift, 1, 247-256.

Touhami, I., Chirino, E., Andreu, J.M., Sánchez, J.R., Moutahir, H., Bellot, J., 2015, Assessment of climate change impacts on soil water balance and aquifer recharge in a semiarid region in south east Spain: Journal of Hydrology, 527, 619-629.

UNESCO (Organización de las Naciones Unidas para la Educación, la Ciencia y la Cultura), 2012, Managing Water under Uncertainty and Risk: París, The United Nations World Water Development Report 4, 407 pp.

Wada, Y., Van Beek, L.P.H., Bierkens, M.F.P., 2012, Nonsustainable groundwater sustaining irrigation: A global assessment: Water Resources Research, 48(6), 1-18.

Wanders, N., Van-Lanen, H.A.J., 2015, Future discharge drought across climate regions around the world modelled with a synthetic hydrological modelling approach forced by three general circulation models: Natural Hazards and Earth System Sciences, 15, 487-504.

White, M.J., Harmel, R.D., Arnold, J.G., Williams, J.R., 2012, SWAT-Check: A Screening Tool to Assist Users in the Identification of Potential Model Application Problems: Journal of Environmental Quality, 43(1), 208-214.

Winchell, M., Srinivasan, R.S., Di Luzio, M., Arnold, J.G., 2013, ArcSWAT Interface for SWAT2012. User's Guide: Blackland Research Center, Texas AgriLife Research, College Station, $464 \mathrm{pp}$.

WRB, 2014, World Reference Base for Soil Resources 2014, International soil classification system for naming soils and creating legends for soil maps: Rome, Food and Agriculture Organization of the United Nations, IUSS Working Group, World Soil Resources Reports, 106, 203 pp.

Manuscrito recibido: noviembre 22, 2017

Manuscrito corregido recibido: julio 4, 2018

Manuscrito aceptado: julio 7, 2018 